\title{
Malfunctioning central venous catheters in children: a diagnostic approach
}

\author{
Alex Barnacle • Owen J. Arthurs • Derek Roebuck • \\ Melanie P. Hiorns
}

Received: 22 July 2007 / Accepted: 29 July 2007 / Published online: 12 October 2007

(C) Springer-Verlag 2007

\begin{abstract}
Central venous access is increasingly becoming the domain of the radiologist, both in terms of the insertion of central venous catheters (CVCs) and in the subsequent management of these lines. This article seeks to provide an overview of the CVC types available for paediatric patients and a more detailed explanation of the spectrum of complications that may lead to catheter malfunction. A standard catheter contrast study or 'linogram' technique is described. The normal appearances of such a study and a detailed pictorial review of abnormal catheter studies are provided, together with a brief overview of how information from catheter investigations can guide the management of catheter complications.
\end{abstract}

Keywords Central venous catheter - Complications . Children

\section{Introduction}

Central venous access forms a vital part of the management of many paediatric conditions. For many years, tunnelled central venous catheters were largely reserved for patients on chemotherapy regimens or long-term total parenteral nutrition (TPN), but more recently, reliable central venous access has proved central to the management of an extensive range of conditions such as those requiring long-term antibiotic therapy, chemotherapy or haemodialysis. The

A. Barnacle $\cdot$ O. J. Arthurs $\cdot$ D. Roebuck $\cdot$ M. P. Hiorns $(\square)$ Radiology Department, Great Ormond Street Hospital,

Great Ormond Street,

London WC1N 3JH, UK

e-mail: hiornm@gosh.nhs.uk emergence of image-guided vascular access techniques has brought central venous access increasingly into the domain of interventional radiology (IR), with perceived advantages of shorter operating times, an increased likelihood of achieving access in difficult cases, fewer procedural complications, lower operating costs, and a probable improvement in longterm venous patency rates [1-10]. This shift from general surgery to radiology has, however, brought with it an increased demand on radiology departments to maintain and manage indwelling central venous catheters (CVCs). In children, meticulous attention to catheter position is vital to ensure that CVCs are kept functioning for as long as possible in order to minimize the number of catheter replacements a child may have to undergo. In some practices, malfunctioning catheters are removed and replaced without investigation; in other centres, including our institution, it is recognized that there are a number of interventions available to salvage blocked or displaced catheters, allowing alternative access sites to be preserved for future use. Plain radiographs and fluoroscopic contrast examinations are central to the radiological investigation of malfunctioning catheters [11, 12]. Many radiologists, however, may be perplexed by the increasingly wide variety of venous access devices available and unaware of some of the potential CVC-related complications that develop in children or the catheter-salvage techniques available.

This article gives a brief overview of the central venous access devices available and the potential causes of catheter malfunction. A standard catheter contrast study or 'linogram' technique is described. The normal appearances of such a study and a detailed pictorial review of abnormal catheter studies are provided, together with a brief overview of how information from catheter investigations can guide the management of catheter complications. 


\section{Background}

Central venous access is required for the safe delivery of a number of drugs and other intravenous therapies into largecalibre vessels, to allow adequate dilution of the drug and avoid vessel irritation. Here the term 'central' is taken to encompass the brachiocephalic veins, the superior vena cava (SVC), the suprahepatic inferior vena cava (IVC) and the right atrium (RA). A centrally placed catheter with a reasonable-size lumen also allows regular noninvasive blood sampling, which has significant benefits for both the clinical team and the patient.

There is much debate surrounding the issue of the ideal CVC tip position and a detailed presentation of this argument is beyond the remit of this article. Suffice to say that the argument that a catheter tip position at the level of the RA is unsafe due to the risk of myocardial perforation and subsequent tamponade is increasingly unsupported in modern practice with the advent of softer catheter tips, with the probable exception of $2 \mathrm{~F}$ catheters, traditionally inserted by the cot-side as neonatal long lines, which have a slightly stiffer tip. There is much in the literature regarding cardiac perforation by $2 \mathrm{~F}$ neonatal long lines in low-birth-weight infants and it seems generally accepted that these catheters are safer when positioned outside the pericardial reflections [13-20]. This paper, however, deals specifically with radiologically or surgically inserted Silastic (silicone elastomer) catheters of $4 \mathrm{~F}$ calibre and above. It is now recognized that these larger-calibre catheters are significantly less likely to develop tip thrombus or a fibrin sheath when left to move freely within fast-flowing blood at the level of the right atrium and are likely to function for a significantly longer period of time in this position [8,21-25]. The position of the RA is best estimated at a level 1.5 vertebral bodies below the carina on a chest radiograph [26, 27]. It is worth remembering, however, that the position of a catheter tip will vary widely with patient positioning, particularly in children, and should be evaluated with caution on any image $[8,22,28-30]$.

\section{Catheter types}

There is a vast array of central venous access devices available for paediatric use; particular devices may be preferred in different centres. In general, however, catheter subtypes can be defined according to their site of insertion, whether they are placed via a subcutaneous tunnel or, with particular reference to haemodialysis catheters, according to function. To achieve a central tip position, a catheter can be inserted using a tunnelled or a nontunnelled technique. Tunnelled devices are usually inserted via access to a neck vein and are tunnelled to that site through the adjacent subcutaneous tissues, exiting the skin at an appropriate position on the chest wall. The tunnelled, extravascular portion of the catheter is considered to provide a barrier to ascending infection and with time will adhere to the subcutaneous tissues, decreasing the likelihood of catheter displacement. This adherence is encouraged by the presence of a short Dacron cuff attached to the catheter, which causes a focal region of fibrosis or ingrowth in the track and thus provides further anchorage for the catheter (Fig. 1). Insertion of such catheters almost always requires general anaesthesia in children to allow safe venous puncture. The vein puncture itself can be performed using a percutaneous, US-guided technique or a surgical open venotomy. Removal of a cuffed catheter that has been in situ for longer than a week or so generally also requires a general anaesthetic in children, as removal or dissection of the cuff can be traumatic. Hence, tunnelled CVCs are best suited for medium- to long-term venous access requirements such as chemotherapy and TPN regimens. Tunnelled catheters range in calibre from $2.7 \mathrm{~F}$ to $12 \mathrm{~F}$ and have a single or double lumen. Haemodialysis catheters are very similar to other double-lumen catheter devices. The lumens are generally of a larger calibre, however, to allow high flow rates for efficient dialysis and the end holes or tips may be staggered (Fig. 2).

An alternative to a traditional tunnelled CVC is a totally implanted CVC or implanted venous access device (iVAD), commonly known as a portacath. This comprises a reservoir attached to a standard Silastic catheter (Fig. 3). The catheter is inserted using a standard tunnelled catheter technique. The reservoir is buried within the subcutaneous tissues of the chest wall, so that the device is completely implanted. When required, the reservoir is accessed percutaneously via a specialised noncoring needle. The reservoir can be accessed up to 1,000 times before replacement is needed. Such buried devices have a lower infection rate than externalized catheters and allow greater freedom for social activities such as swimming, but require patients to undergo

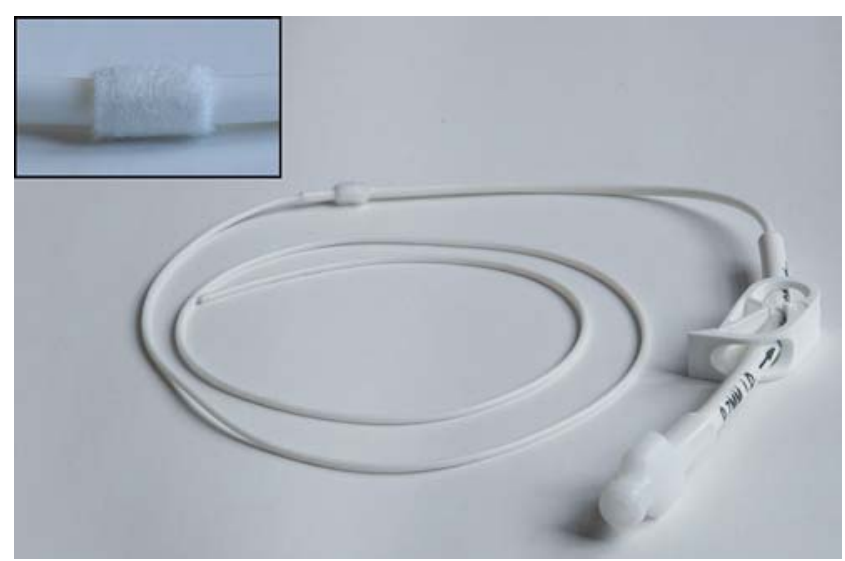

Fig. 1 A 4F single-lumen Hickman (Bard Access Systems, Salt Lake City, Utah) catheter for tunnelled central venous access. Inset illustrates the Dacron ingrowth cuff 


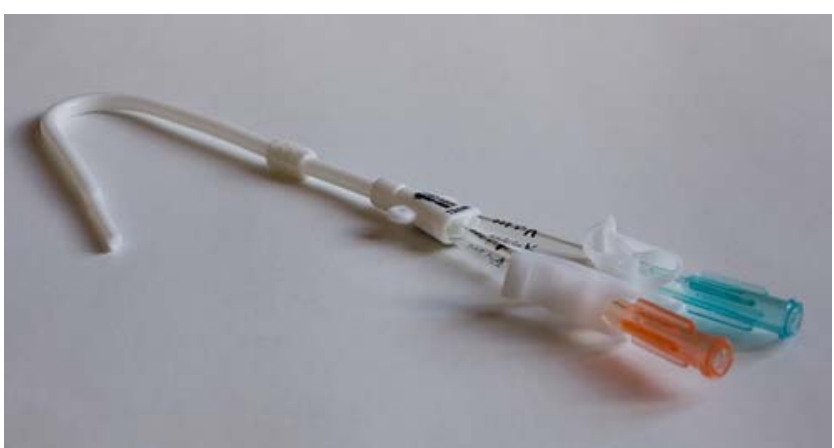

Fig. 2 A permanent (tunnelled) haemodialysis catheter with staggered tip for optimal flow rates (12.5F Vas-Cath Soft Cell; Bard Access Systems, Salt Lake City, Utah)

repeated needle access procedures. They are best suited for intermittent therapies such as enzyme infusions in metabolic disorders, factor infusions in haemophilia or recurrent antibiotic requirements for conditions such as cystic fibrosis.

Nontunnelled CVCs are placed directly into a vein via a skin incision overlying the vein and are simply sutured to the adjacent skin (Fig. 4). Access sites include the neck and the extremities. Nontunnelled neck lines are commonly placed for complex anaesthesia cases or in the intensive care setting. The neck veins can also be used for short-term dialysis catheters in patients who do not require a longerterm tunnelled device. For longer-term indications for a nontunnelled line, operators usually prefer to access venous sites in the extremities. Small-calibre (4-5F) peripherally inserted central catheters (PICCs) are usually placed via the veins of the upper arm (Fig. 5). In a cooperative child they can often be placed via a peripheral venous cannula, without sedation or general anaesthesia, and are most useful for providing reliable access for medium-term therapies in children. The catheter is advanced to the level of the central vessels using fluoroscopic guidance in the radiology department or a non-image-guided 'best-estimate' technique at the bedside. These catheters provide reliable venous access for indications such as extended antibiotic

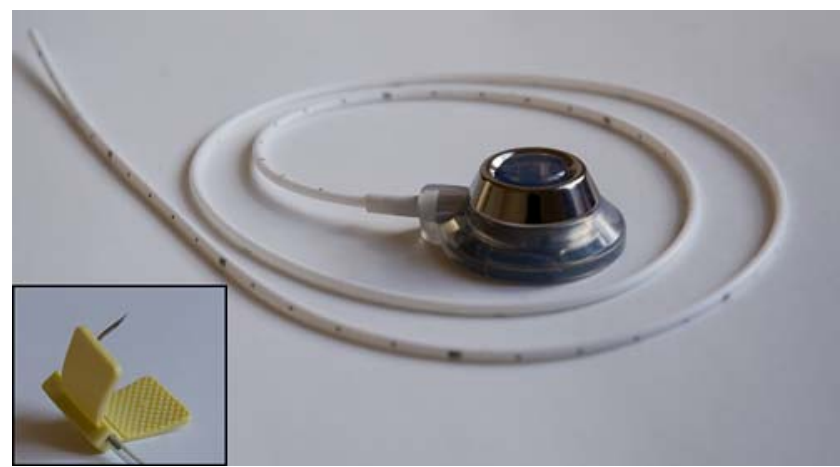

Fig. 3 A totally implantable port (Bard Access Systems, Salt Lake City, Utah) with reservoir and $6.6 \mathrm{~F}$ catheter. Inset illustrates the specialized noncoring port access needle

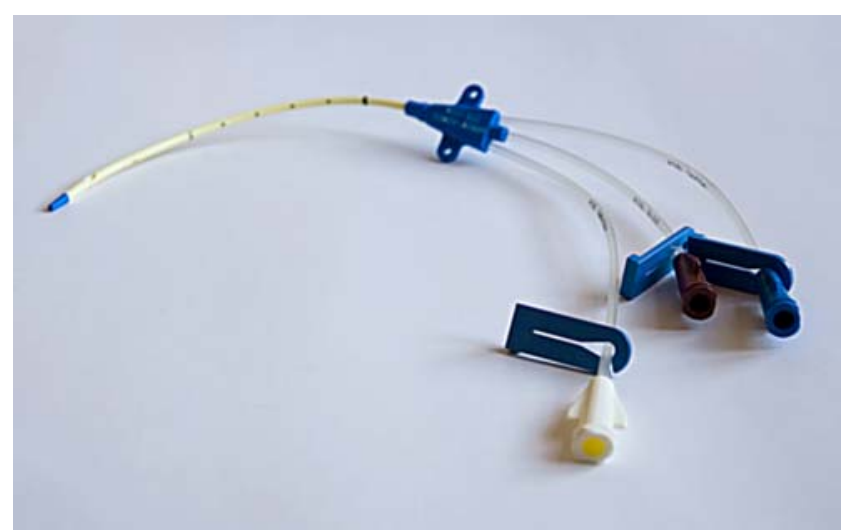

Fig. 4 A triple-lumen 7F catheter (Arrow-Howes; Arrow International, Reading, Pa.) for short-term nontunnelled central venous access

treatment in patients with osteomyelitis or infected neurosurgical shunts and for repeated blood-sampling in needlephobic patients, or children in whom peripheral access is becoming technically difficult. PICCs are also increasingly used for longer-term therapies such as chemotherapy, pulmonary hypertension and TPN, and can function for a year or more. Single-lumen peripherally inserted CVCs or PICCs are commonly $4 \mathrm{~F}$ in calibre; this allows more reliable function than the $2 \mathrm{~F}$ neonatal long lines, particularly for blood sampling (aspiration via a catheter requires a faster flow rate in the vessel being sampled, and is therefore more sensitive to lumen calibre when compared with infusion through the same catheter). Double-lumen $5 \mathrm{~F}$ catheters are also widely used, although each lumen is small and hence these catheters, although useful for multidrug therapies, are less reliable for blood sampling. Some types of PICC are now approved for CT contrast medium infusions via a pump injector.

\section{Strategies in managing catheter malfunction}

CVC malfunction can be approached by the clinical team and the radiologist in a number of ways. In some centres a

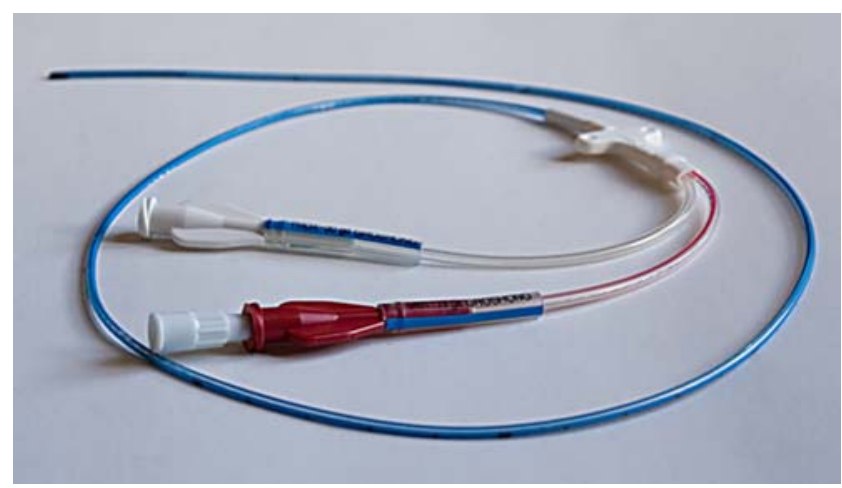

Fig. 5 A double-lumen 5F PICC (Bard Access Systems, Salt Lake City, Utah) 
policy of removing all malfunctioning catheters without investigating the cause of the malfunction is maintained. In most institutions, however, protocols are available for attempted thrombolysis of blocked catheters and in some units revision of displaced or incorrectly positioned lines is attempted. In our opinion, an attempt should always be made to diagnose the cause of a malfunctioning catheter, with the aim of possible catheter salvage and, therefore, a potential reduction in the number of both repeated anaesthesia and venous access attempts that a patient may have to undergo. An institutional policy regarding the management of malfunctioning CVCs is helpful and should be made with the facilities of that institution in mind. In particular, contrast studies involving radiation exposure should only be undertaken if the results of the study would alter the subsequent management of the line. In general, an IR department is more likely to be able to offer salvage procedures than a surgically managed service, due to differences in both the imaging and interventional techniques available within each speciality $[3,10,31]$.

\section{Initial examination of the catheter}

It is important to examine both the catheter and its skin exit site prior to performing any radiographic investigation, so that a simple cause of catheter malfunction, such as a break in the catheter itself or wear at the clamp site, can be established. It is not uncommon to find that the skin suture of a newly placed catheter is too tight and is restricting flow.

\section{Fluoroscopic or plain radiographic views}

Plain radiographic views of the catheter should be acquired prior to any contrast-enhanced study. These can be acquired as a chest radiograph or by using low-dose fluoroscopy at the time of a planned contrast investigation. This allows misplaced catheters (Fig. 6) and catheters of an incorrect length to be identified (Fig. 7). The entire catheter must be imaged. Studies limited to the intravascular portion of the catheter will frequently miss abnormalities such as kinking of the catheter within the skin, a finding that is particularly common with portacaths (Fig. 8), and looping or twisting of a catheter in the neck (Fig. 9). More than one view may be required to confirm a kink in the catheter of a portacath; angulation of the catheter most commonly occurs at the origin of the subcutaneous tunnel, adjacent to the reservoir (Fig. 10). Occasionally, a portacath reservoir can become detached from its catheter, and children who are compulsive 'port fiddlers' can encourage a catheter to loop around the port chamber so that over time the intravascular portion is shortened. A catheter may be accidentally pulled back or may migrate backwards into the soft tissues of the neck, so that the tip lies outside the vein entirely. This second complication seems to occur most commonly in chubby infants with redundant tissue in the neck (Fig. 11). Despite accurate initial placement, catheter tips can flick into an
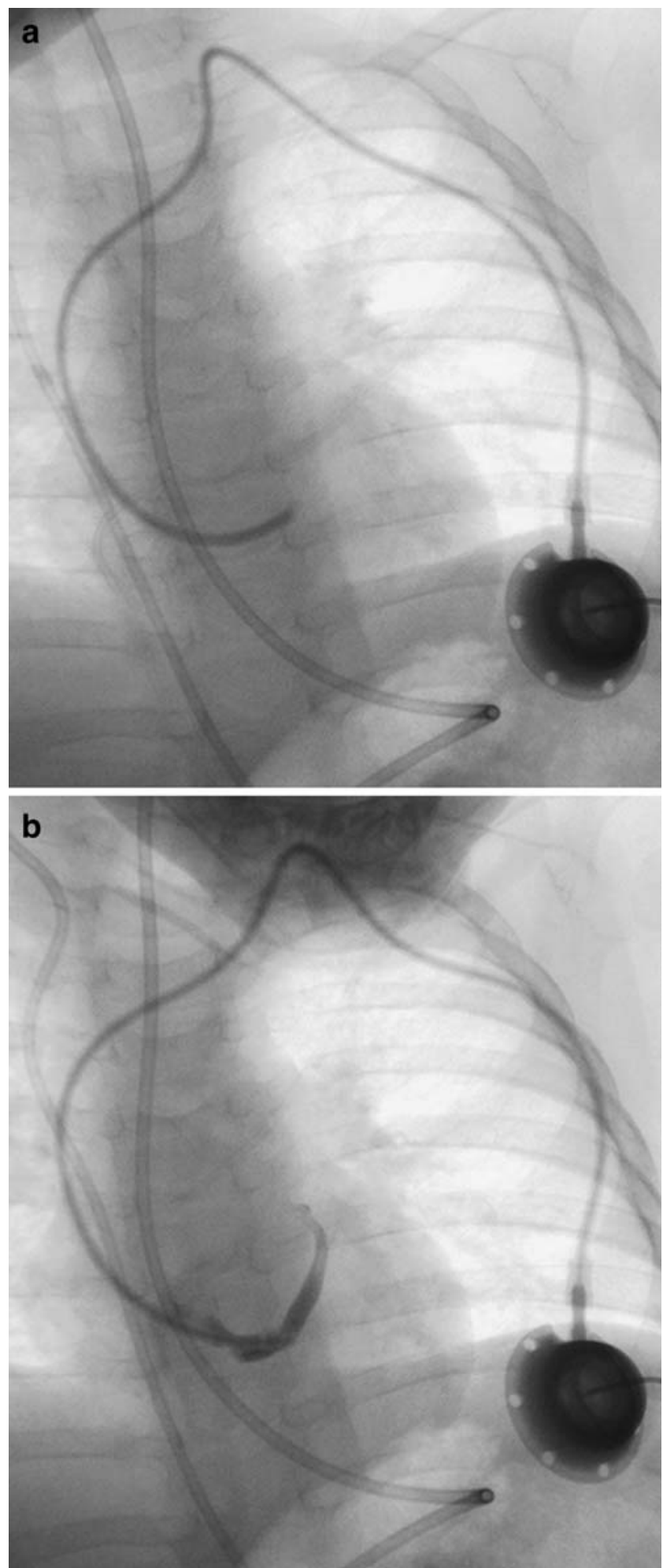

Fig. 6 Misplaced catheter. a Fluoroscopic image of a 10-month-old male with a portacath in situ, the tip of which has been placed into the coronary sinus. $\mathbf{b}$ The position of the tip is confirmed by a contrast medium study 


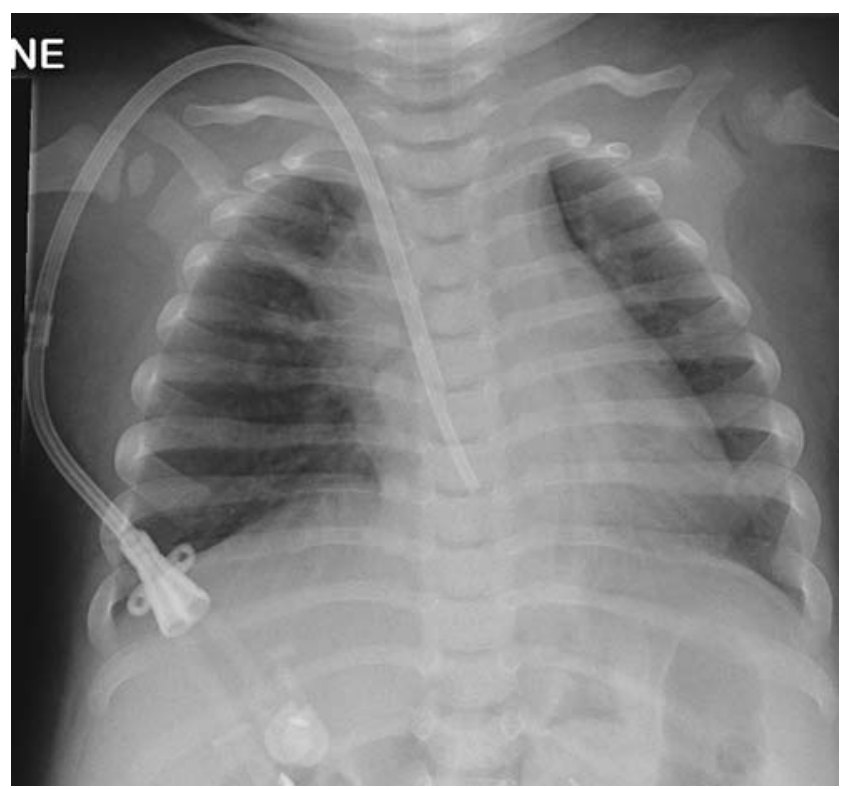

Fig. 7 A chest radiograph in a 13-month-old male with a permanent haemodialysis catheter, the tip of which lies at or beyond the level of the tricuspid valve causing intermittently poor flow rates

incorrect position with changes in posture or intrathoracic pressure; this occurs more commonly with catheter tips placed at the level of the SVC rather than the RA. The commonest site for a catheter tip to migrate to is the ipsilateral internal jugular vein or the contralateral brachiocephalic vein (Fig. 12). Observing the catheter tip during fluoroscopic screening, even before injecting contrast medium, allows the operator to determine whether the tip is moving freely or is wedged against a vessel or chamber wall. Exaggerated movement of the catheter when in an intracardiac position is strongly suggestive of malposition of the catheter tip within the right ventricle; most operators would agree that such a line should be shortened or replaced in such a situation, even if the child is asymptomatic, due to the risk of catheter-induced arrhythmias. Fluoroscopic screening should be performed using a low pulse rate (three pulses per second) and where available, the image-grab facility should be used to minimize radiation exposure.

\section{Performing a contrast study}

If the initial examination of the line is unremarkable, a contrast-enhanced study ('linogram') should be performed. The catheter should be accessed using a sterile technique and, where possible, any heparin solution dwelling in the line should be aspirated and discarded. Inability to aspirate from the catheter should be documented, as this narrows the list of potential causes of catheter malfunction, as discussed below. If only one lumen of a multilumen catheter is malfunctioning, imaging should first be performed through the functioning lumen as this may demonstrate the under- lying problem and obviate the need for access of the second lumen. Water-soluble nonionic contrast medium should be used and the operator should aim to minimize the dose of contrast medium that the child receives.

The initial contrast medium injection should be performed slowly while the entire catheter is screened so that complications anywhere along the length of the line are readily identified (Fig. 13). The operator should look for signs of pooling of contrast medium in the soft tissues surrounding the catheter. Extravasation and pooling of contrast medium in the neck are usually painful; a slow gentle injection will minimize both the patient's discomfort and the risk of loss of the child's confidence in the operator. When examining a malfunctioning portacath device, tangential and en face views of the port reservoir should be obtained, to ensure that it fills uniformly and does not
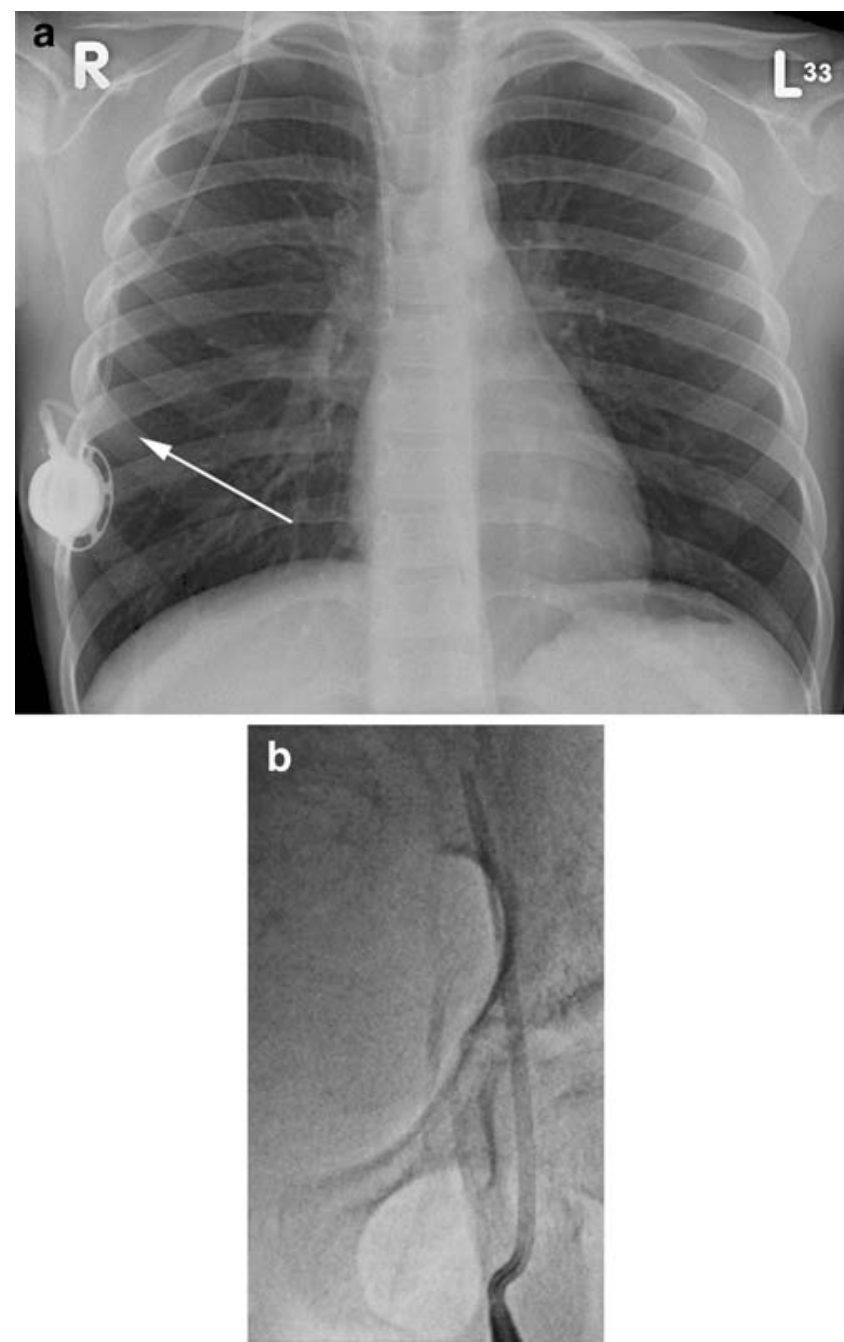

Fig. 8 Kinking of the catheter. a Chest radiograph of a 6-year-old male with a portacath in situ. The catheter is kinked just beyond the reservoir (arrow). b Fluoroscopic image of a femoral temporary dialysis catheter in a 15-year-old male. The catheter is kinked at the vessel entry site, causing poor flow rates 


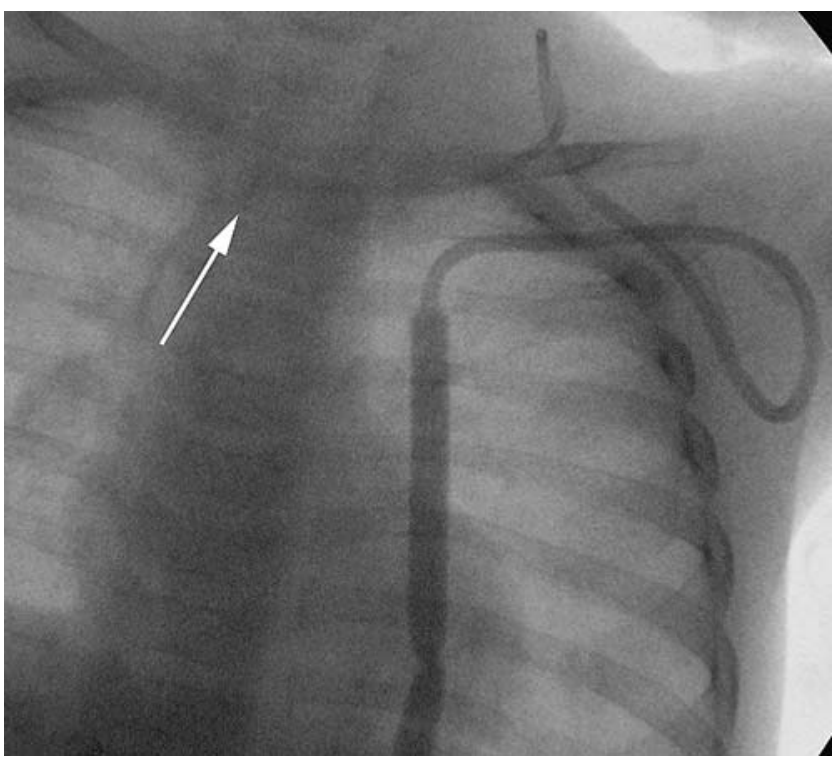

Fig. 9 Fluoroscopic image of a tunnelled catheter in a 2-year-old child. The catheter has twisted in the soft tissues of the neck, causing the catheter tip to be partially withdrawn (arrow)

contain thrombus (Fig. 14). If the initial slow injection of contrast medium appears normal, a controlled but forceful hand injection should then be performed to study in more detail the flow of contrast medium away from the catheter tip. Digital subtraction angiography (DSA) should be used during image acquisition where possible, to achieve a diagnostic study with one injection, minimizing both contrast agent and radiation dose. Cooperative patients should be encouraged to breath-hold. If the study is performed in an imaging suite without DSA capability, screening should be focused on the catheter tip and the fluoroscopic pulse rate may have to be increased to either 15 pulses per second or 'continuous' screening to obtain adequate images. Diagnostic imaging of the contrast medium jet is hardest in small patients with rapid breathing and significant cardiac motion.

The contrast medium jet should be of the same diameter as the lumen of the catheter and should exit the catheter in a linear forward direction, rapidly moving away from the catheter tip rather than pooling at the tip. The jet should fan out appropriately to fill the lumen of the vessel or chamber (Fig. 15). The operator should be aware of the configuration of the catheter, so that a catheter with a side hole, such as the Groshong PICC (Bard Access Systems, Salt Lake City, Utah) is not misinterpreted as a fractured or partially occluded line (Fig. 16).

It is important to remember to study both lumens of a double-lumen catheter if injection of the first lumen is normal. In patients with intermittent catheter malfunction, attempts should be made to recreate the situation in which problems occur; often, catheter function is position-dependent and placing the child in the troublesome position may demon- strate the cause of catheter malfunction. This may include a significant change in tip position following a change in posture or intermittent occlusion of the catheter lumen at the level of the clavicle due to catheter compression between the clavicle and the first rib ('pinch-off' syndrome).

If the child presents with symptoms of venous occlusion, such as localized pain and swelling over the venous access site or sudden swelling of the face or extremity, a formal venogram via a peripheral cannula can be performed to determine the presence of catheter-related venous thrombosis (see below).

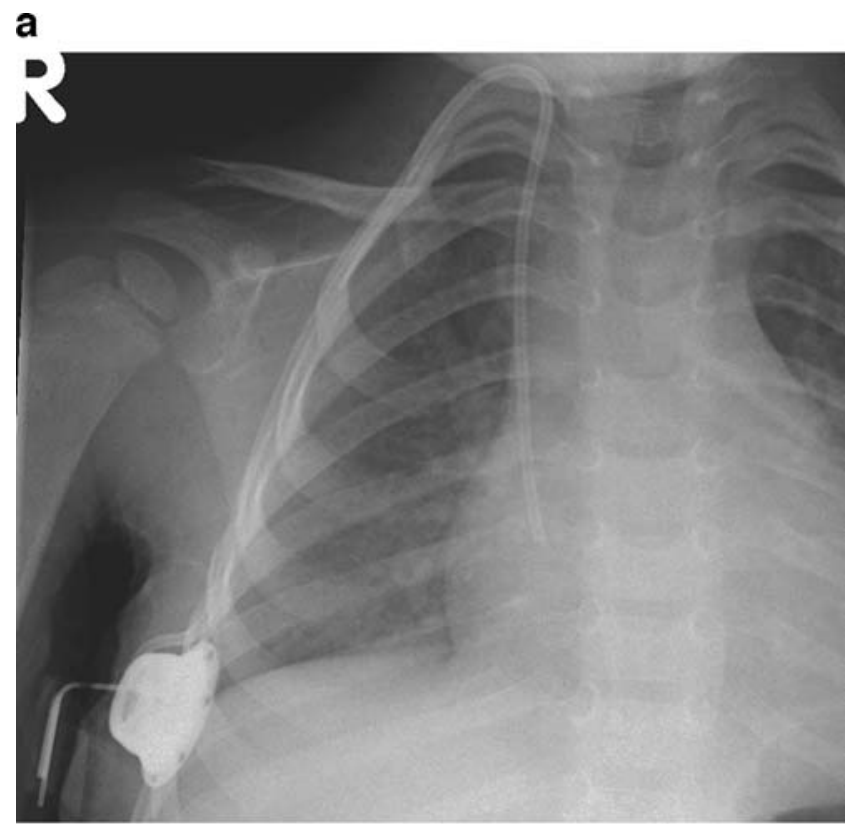

b

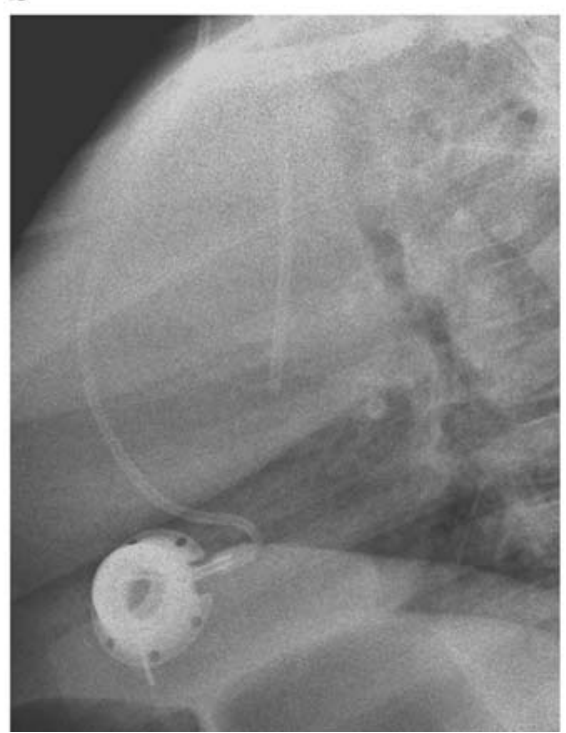

Fig. 10 Kinking of the catheter. a, b AP (a) and lateral (b) views of a portacath in a 2-year-old female demonstrating sharp angulation of the proximal catheter, limiting infusion flow rates. The kink is not clearly seen on the AP view 


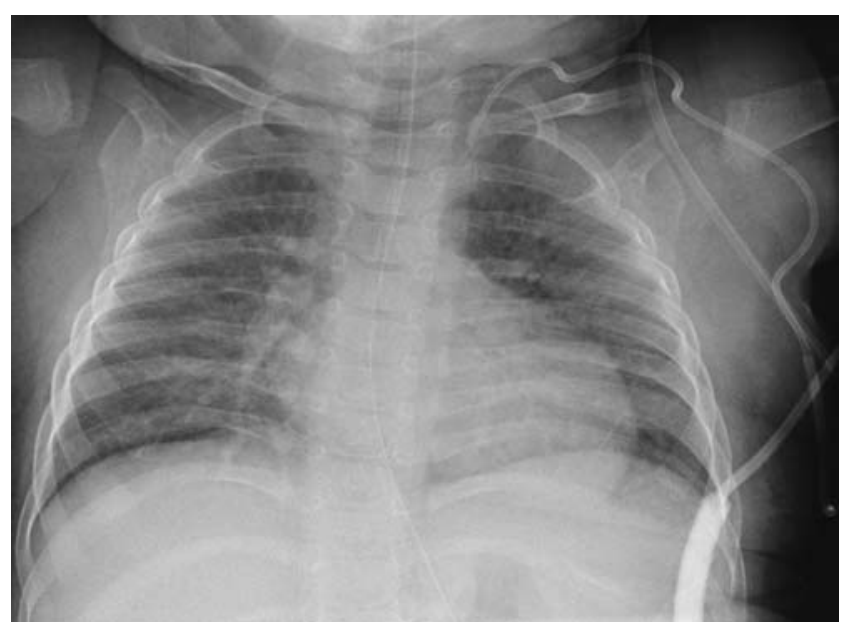

Fig. 11 Chest radiograph of a 9-month-old infant with a tunnelled CVC in situ. The catheter has migrated back into the redundant soft tissues of the neck and chest wall

Once the study is completed, the catheter should always be flushed and, where required, 'locked' with heparin solution of an appropriate concentration. This should be documented in the clinical notes as part of the examination. If the catheter is deemed unsafe for use, this too should be clearly documented.

\section{Interpreting an abnormal contrast study}

Once contrast medium has been instilled, a careful examination of the entire length of the catheter should be performed. Abnormal findings may be subtle, such as narrowing of the lumen at the site of the skin suture or clamp (Fig. 17). Contrast medium will leak into the soft tissues at the site of any break in the catheter wall (Fig. 18). In such cases the point of extravasation can often be clinically predicted by the patient's clinical symptoms (focal swelling within the subcutaneous tunnel or leak of infusate from the catheter exit site) and the contrast agent dose therefore minimized.

Inability to aspirate from a catheter that otherwise flushes well indicates one of three possible causes of catheter malfunction: the presence of a fibrin sheath, catheter tip thrombus, or position of the catheter tip against a vessel or chamber wall. All three causes create a valve effect at the catheter tip, so that the obstruction is temporarily alleviated by forward pressure during infusion but recurs with negative pressure on attempted aspiration (Fig. 19).

Gentle contrast medium injection through a catheter that is resting against a vessel or chamber wall will demonstrate rapid but asymmetric or oblique flow of contrast agent away from the catheter tip (Fig. 20). This appearance should be distinguished from an irregular jet due to tip thrombus (see below). It is vital to identify this problem during an initial slow instillation of contrast medium as a forceful injection may damage the vessel wall.

A fibrin sheath or sleeve thrombus is caused by build-up of fibrin material around the intravascular portion of the catheter. This phenomenon may occur in the majority of catheters, as early as $24 \mathrm{~h}$ after catheter insertion [8, 32, 33]. Fibrin sheaths cause malfunction only when the sheath extends around or over the tip of the catheter. A complete sheath prevents any contrast medium from flowing away

\section{a}

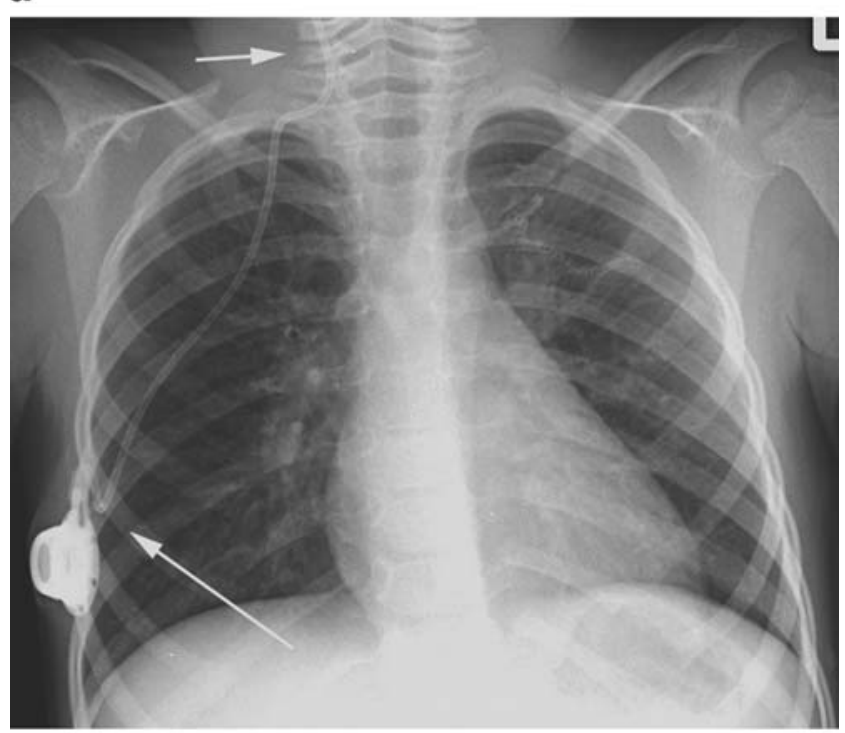

b

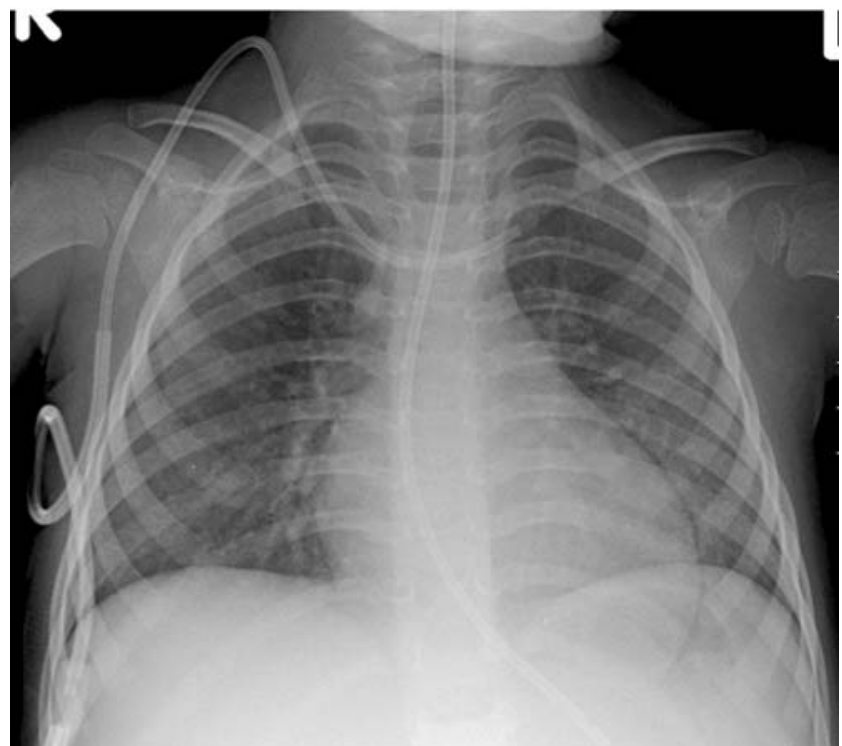

Fig. 12 Migration of the catheter tip. a Chest radiograph of a 4-yearold male with a portacath in situ. The catheter tip lies in the right internal jugular vein (short arrow). Note that the proximal catheter is also kinked (long arrow). b Chest radiograph of a 2-year-old female with a tunnelled CVC in situ. The catheter tip lies in the left brachiocephalic vein 


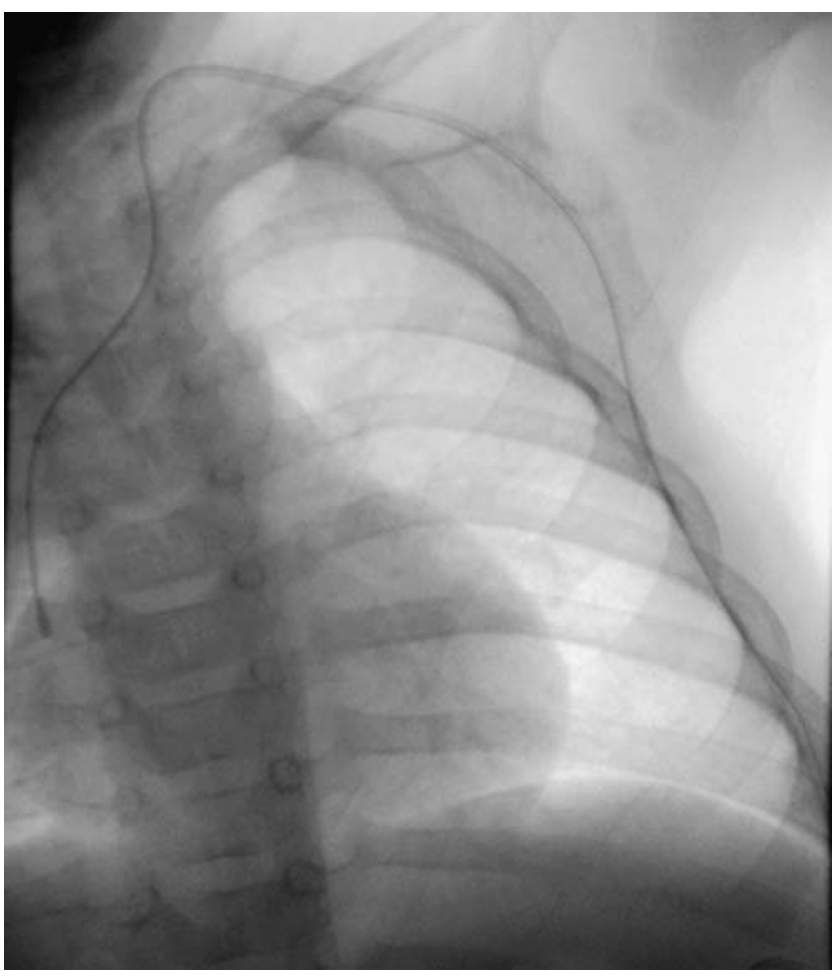

Fig. 13 Fluoroscopic imaging of a tunnelled catheter in a 4-monthold female. The entire catheter is imaged during slow instillation of contrast medium

from the catheter tip; instead it tracks retrogradely along the intravascular portion of the catheter, between the catheter and the fibrin sheath, and then spills into the soft tissues at the point of venous access (Fig. 21). In such cases, the patient will present with symptomatic swelling at the venous access site, usually the neck, during attempted catheter flushes. It is essential to identify initial contrast medium pooling at the catheter tip in the early phase of the injection followed by tracking of contrast medium back along the length of the catheter on later images, so that this is not misinterpreted as extravasation due to catheter fracture. To ensure a correct diagnosis in such cases, sequential images should be acquired or 'grabbed' during a very slow injection. In some cases, the fibrin sheath may be very thin and it is broken or fenestrated during flushing of the catheter. A circumferential but incomplete fibrin sheath will cause narrowing of the contrast jet as it exits the catheter, with delayed fanning out of contrast medium. This is a subtle, but classic, sign of a fibrin sheath (Fig. 22). The operator must ensure that the contrast agent jet is of a similar diameter to that of the catheter lumen, so that a circumferential thrombus or fibrin sheath causing partial occlusion of the end hole is not missed.

Catheter thrombus is most likely to occur in catheters that are inadequately or infrequently flushed, or that are sited in small vessels with a low volume and rate of blood flow around the tip, as has occurred in the case shown in
Fig. 21. Thrombus within the catheter lumen itself is seen as a filling defect in the catheter during injection (Fig. 23). Tip thrombus causes irregularity of the jet of contrast medium as it exits the catheter and may deflect the jet away
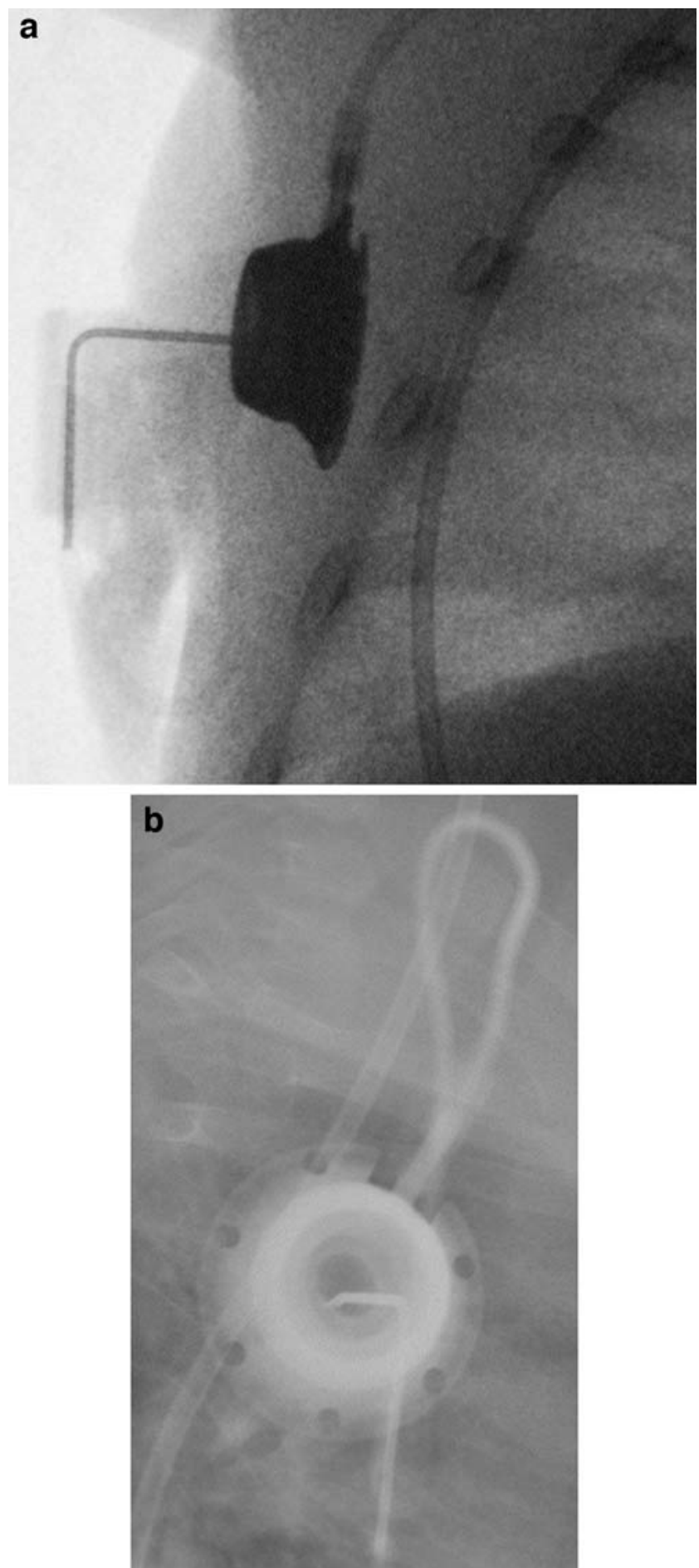

Fig. 14 Normal portacath. a, b Tangential (a) and en face (b) views of a portacath reservoir in a 7-year-old male. Note that in both views there is normal opacification of the reservoir during contrast medium instillation 


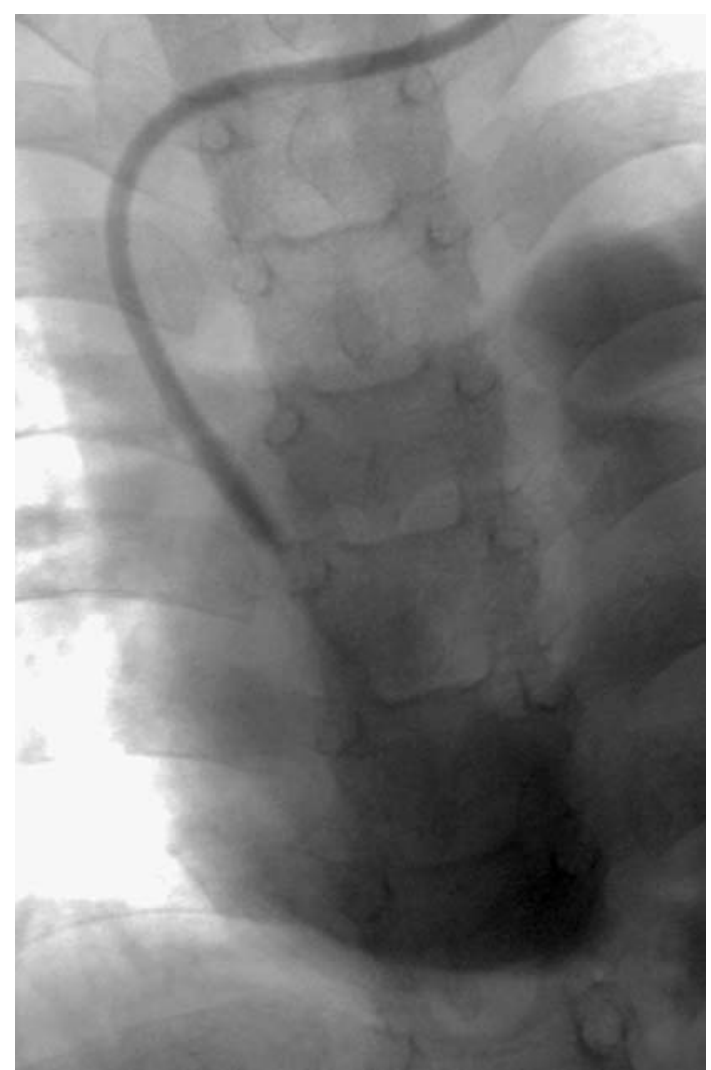

Fig. 15 Fluoroscopic image of contrast medium flowing freely from the catheter tip in an 8-year-old male. The contrast medium immediately fans out to fill the right atrium

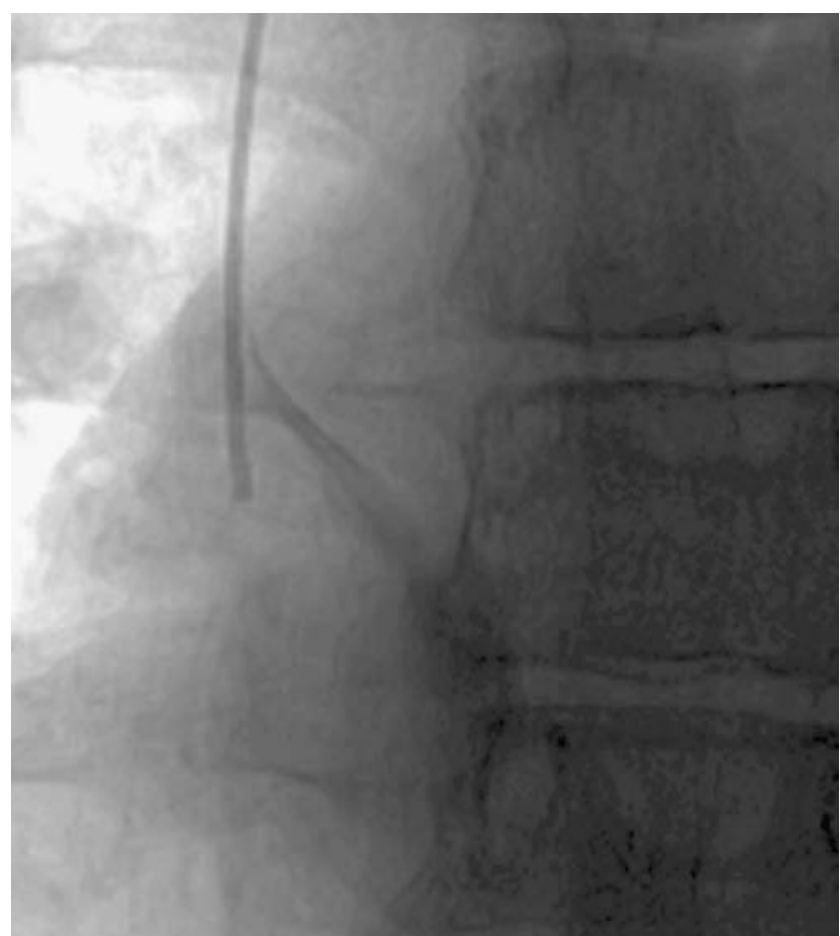

Fig. 16 Normal appearance of the contrast medium jet exiting the side hole of a PICC from the long axis of the catheter (Fig. 24). Thrombus may extend into the vessel itself, which will cause irregular filling of the vessel beyond the catheter tip (Fig. 25).

Catheter-induced venous thrombosis is influenced by catheter dwell time, the size of the catheter relative to the vessel, the access vein used, infusate type, and the presence of any prior venous interventions or systemic comorbidities in the patient [34-38]. It is likely to be a relatively common occurrence [39], although evidence for this in the literature is skewed by variations in the diagnostic techniques and
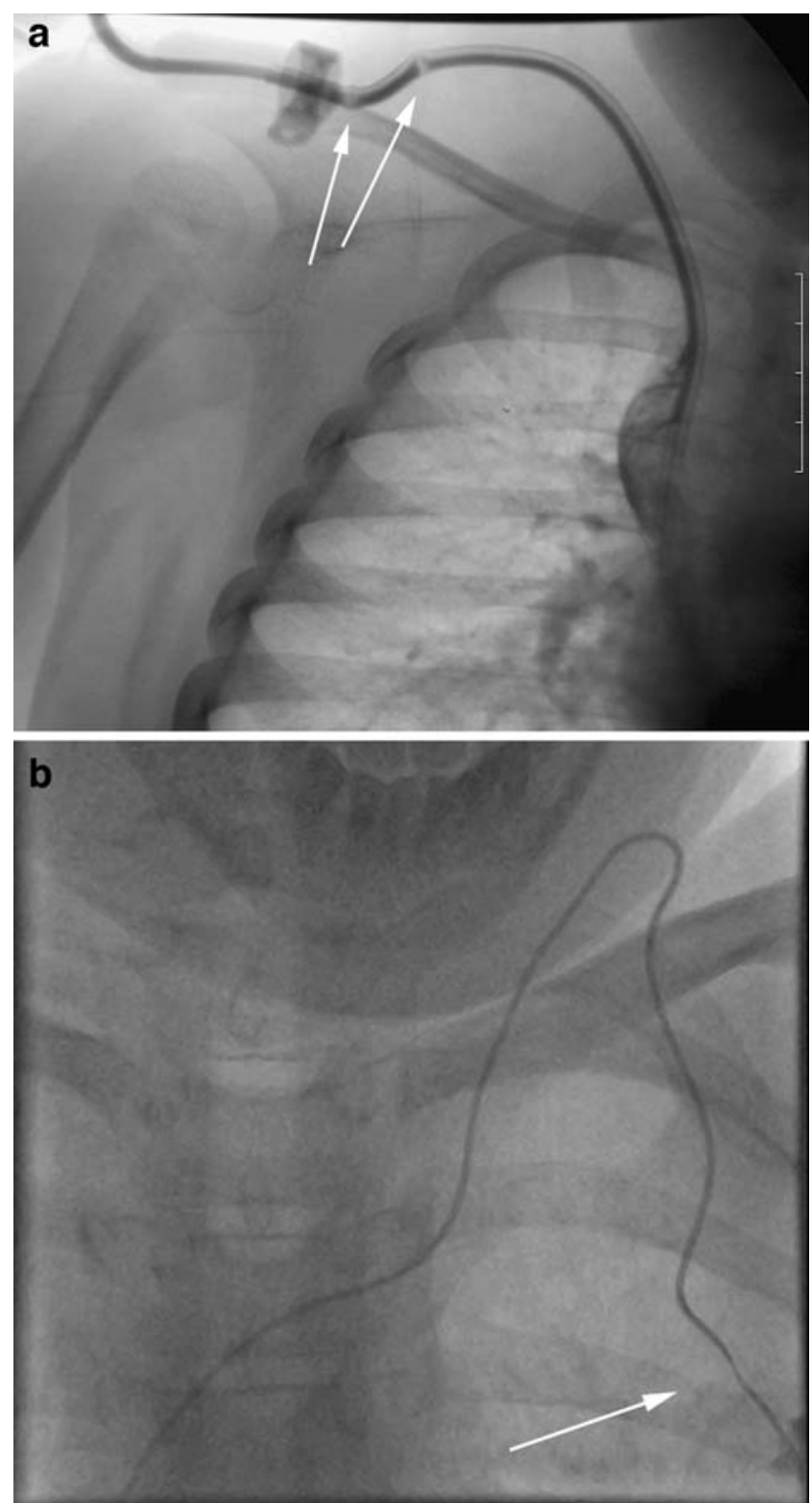

Fig. 17 Catheter narrowing. a Image acquired during contrast injection through a double-lumen catheter in a 7-year-old male. The catheter is damaged at the site of previous kinks or catheter clamps (arrows). b Focal narrowing of the opacified lumen of a PICC (arrow) in a 3-year-old male where the skin suture device has been attached to the catheter too tightly 
pathological definitions used in various studies. Many cases of pericatheter venous thrombosis are asymptomatic and the catheter continues to function, so this complication is likely to be under-reported. Where they occur, symptoms include localized pain and swelling overlying the accessed vein and sudden facial or extremity swelling. It is important that the clinical team rule out septic thrombophlebitis in symptomatic patients. Isolated facial or extremity swelling is, however, strong evidence for acute venous thrombosis [34]. Contrast-

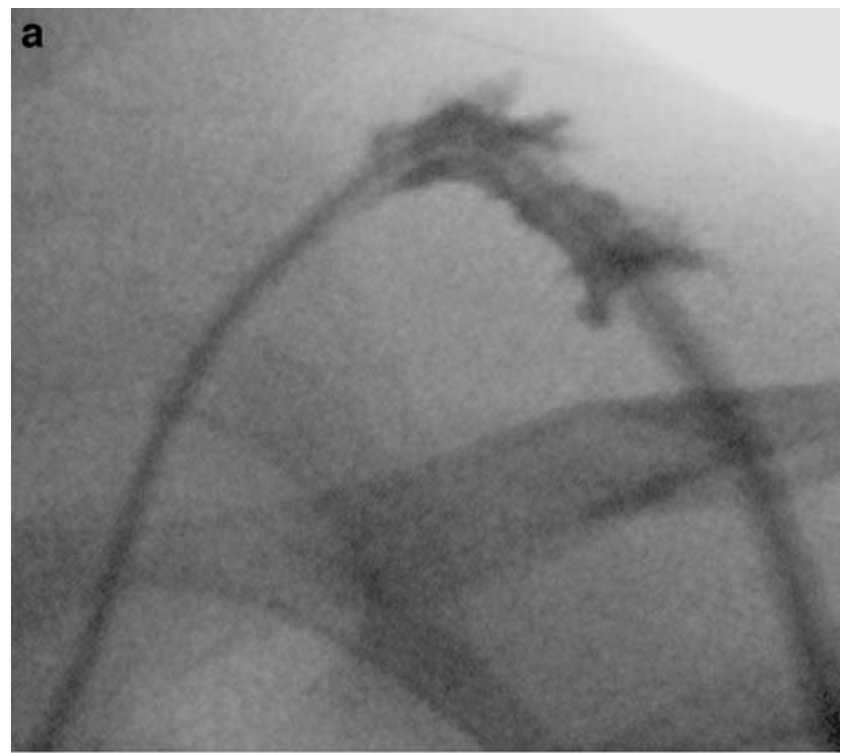

b

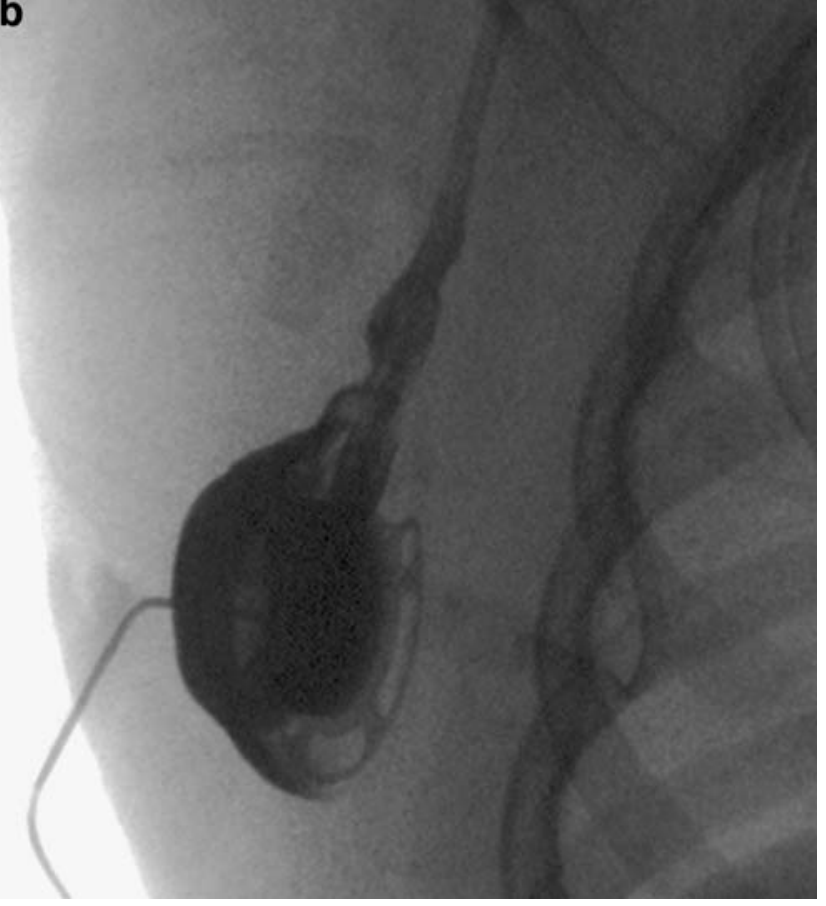

Fig. 18 Extravasation. a Extravasation of contrast medium into the soft tissues at the site of a catheter break. b Contrast medium is seen leaking from a break in the connection between the portacath reservoir and the catheter

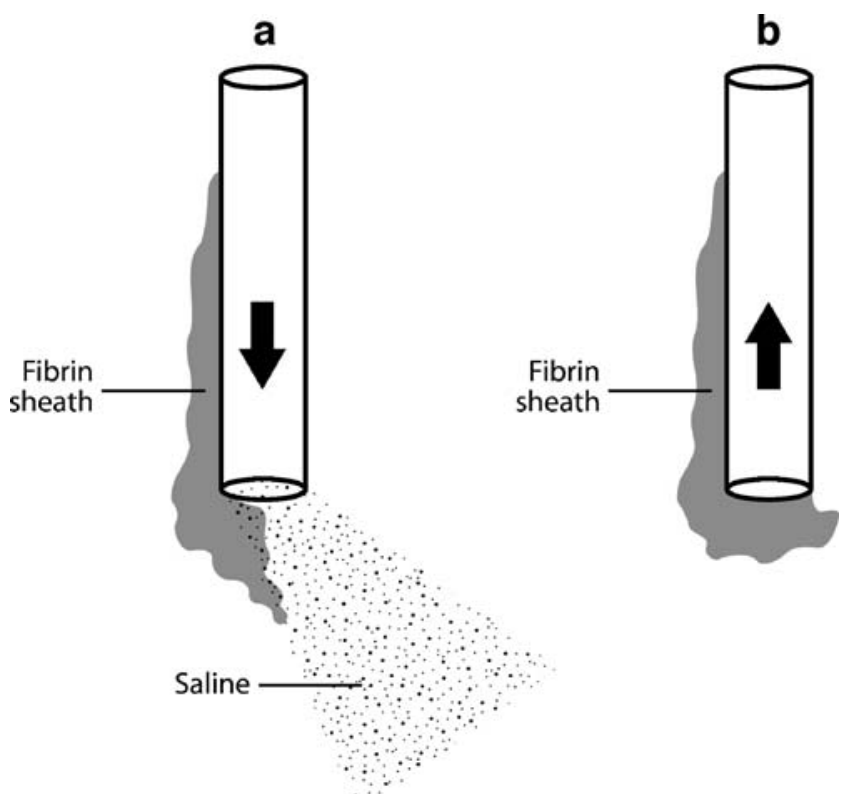

Fig. 19 Line drawing demonstrating the effect of a fibrin sheath. a Flushing the catheter displaces the sheath or thrombus from the catheter tip and allows saline to exit the lumen. b Attempted aspiration sucks the sheath back onto the catheter tip, causing withdrawal occlusion

enhanced catheter studies will demonstrate irregular occlusion of the involved vein (see Fig. 25). In well-established cases, drainage may occur only via tortuous collateral vessels. It may be appropriate to perform a venogram of

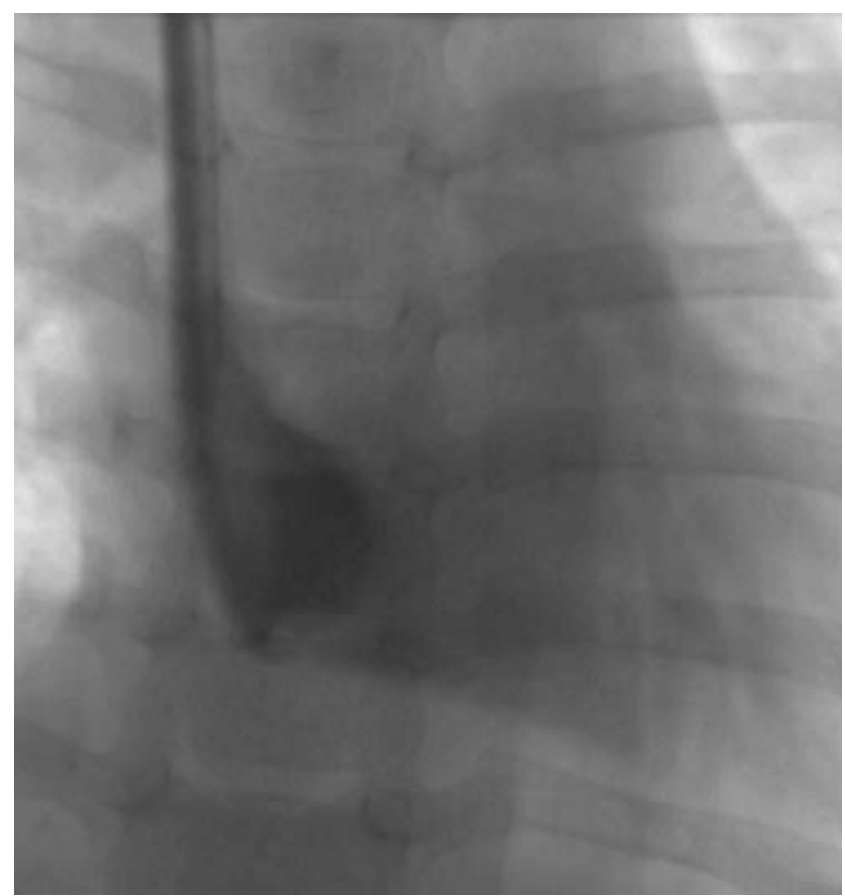

Fig. 20 Fluoroscopic contrast study of a haemodialysis catheter in a 9-year-old female. The lumen with a more distal tip has been opacified. The catheter is too long and the distal tip lies up against the inferior wall of the right atrium, causing the contrast jet to be deflected 
the affected vessel via a peripheral cannula rather than the catheter at this stage, to assess drainage of the extremity (Fig. 26). Some operators may prefer to perform a venogram immediately prior to placement of a new catheter, to allow time for possible recanalization of the vessel or formation of substantial collaterals in the interim period (Fig. 27).

\section{Implications for catheter salvage techniques}

Diagnostic catheter studies are central to the management of malfunctioning catheters. A detailed discussion of catheter salvage techniques is beyond the remit of this review; a number of the techniques outlined below are dependent on the services of a proactive IR department. Most institutions at least have a ward-based thrombolysis protocol for occluded CVCs and these techniques play a significant role in extending the life of a malfunctioning catheter [10, 40-45].

Catheters that are found to be too long can be shortened by a variety of techniques. The simplest involves withdrawal of the intravascular portion of the catheter at the venous access site while the tunnelled portion is left in situ. The catheter is then re-measured and shortened, prior to repuncture of the venous access site and replacement of the intravascular portion of the catheter [46, 47]. If there is sufficient excess length, the catheter can be cut when it is partially withdrawn, and the tip wired to maintain access, avoiding the need for re-puncture.
Fig. 21 Fibrin sheaths. a, b Contrast medium is instilled into a tunnelled catheter in a 4month-old child. The catheter has been partially pulled back and the tip lies within the left brachiocephalic vein. A fibrin sheath occludes the catheter. Instead of flowing away from the catheter tip, contrast medium tracks back along the side of the catheter (a arrow) and extravasates into the tissues of the neck (b). c Contrast study performed in a 2-year-old child with a catheter occluded by a fibrin sheath. Contrast medium tracks back along the catheter and into the subcutaneous tunnel, outlining the cuff, as shown diagrammatically in the line drawing
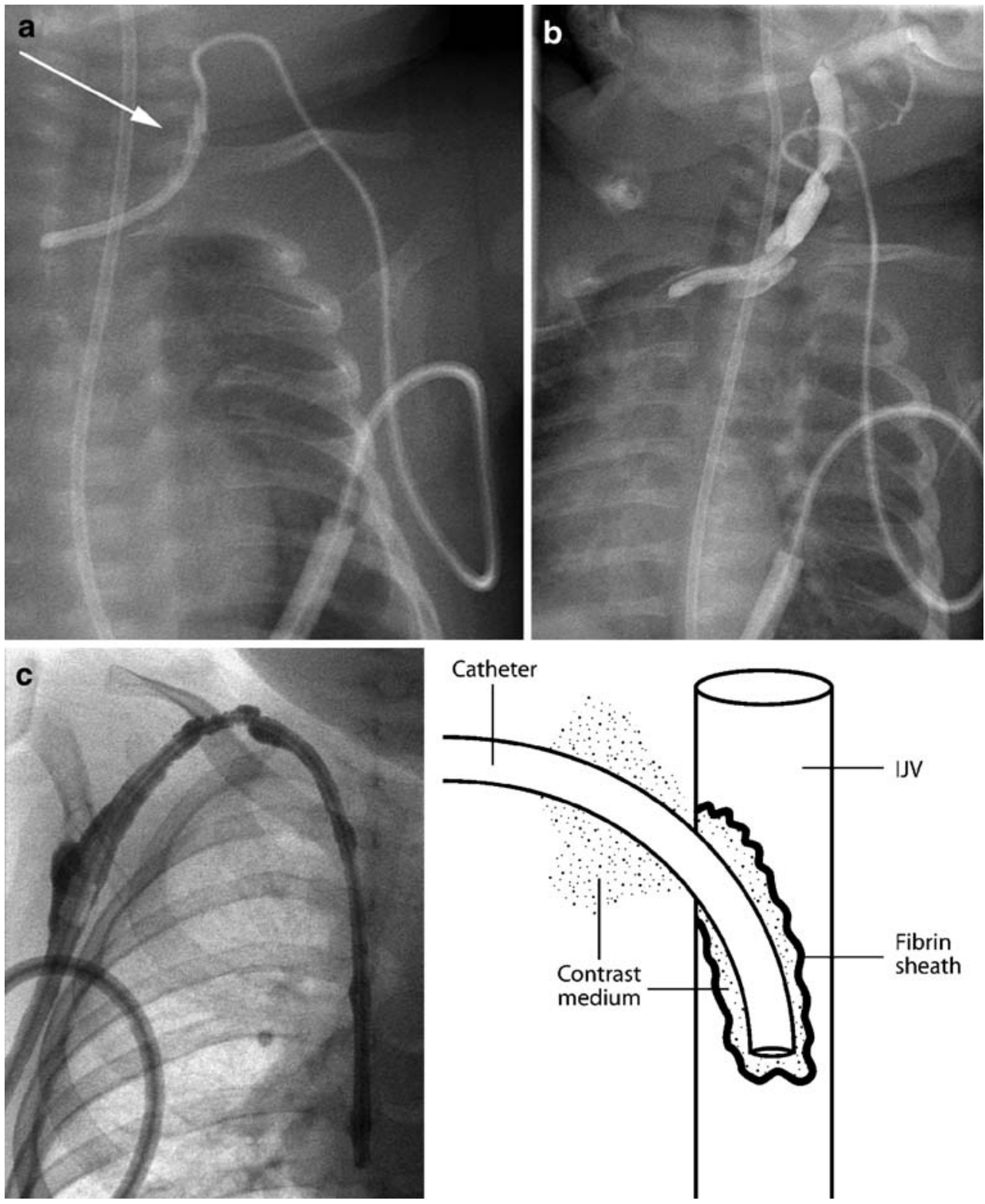
Fig. 22 Fibrin sheaths. a Catheter contrast study in a 5-yearold male. Contrast medium exits the catheter tip in a linear manner, but the contrast jet is significantly narrower than the lumen of the catheter (arrow), implying the presence of a circumferential, nonocclusive fibrin sheath. The effect of the fibrin sheath is shown diagrammatically. b Contrast medium exits the tip of a portacath in an 11-year-old male. The jet fails to fan away from the tip (arrow) of the catheter, instead simply filling the lumen of the sheath. $\mathbf{c}$ A similar appearance is seen during a contrast study of a PICC in another patient. An arrow marks the catheter tip. Note the apparent irregular calibre of the catheter itself, a sign that contrast medium is tracking back along the catheter as shown in Fig. 21c
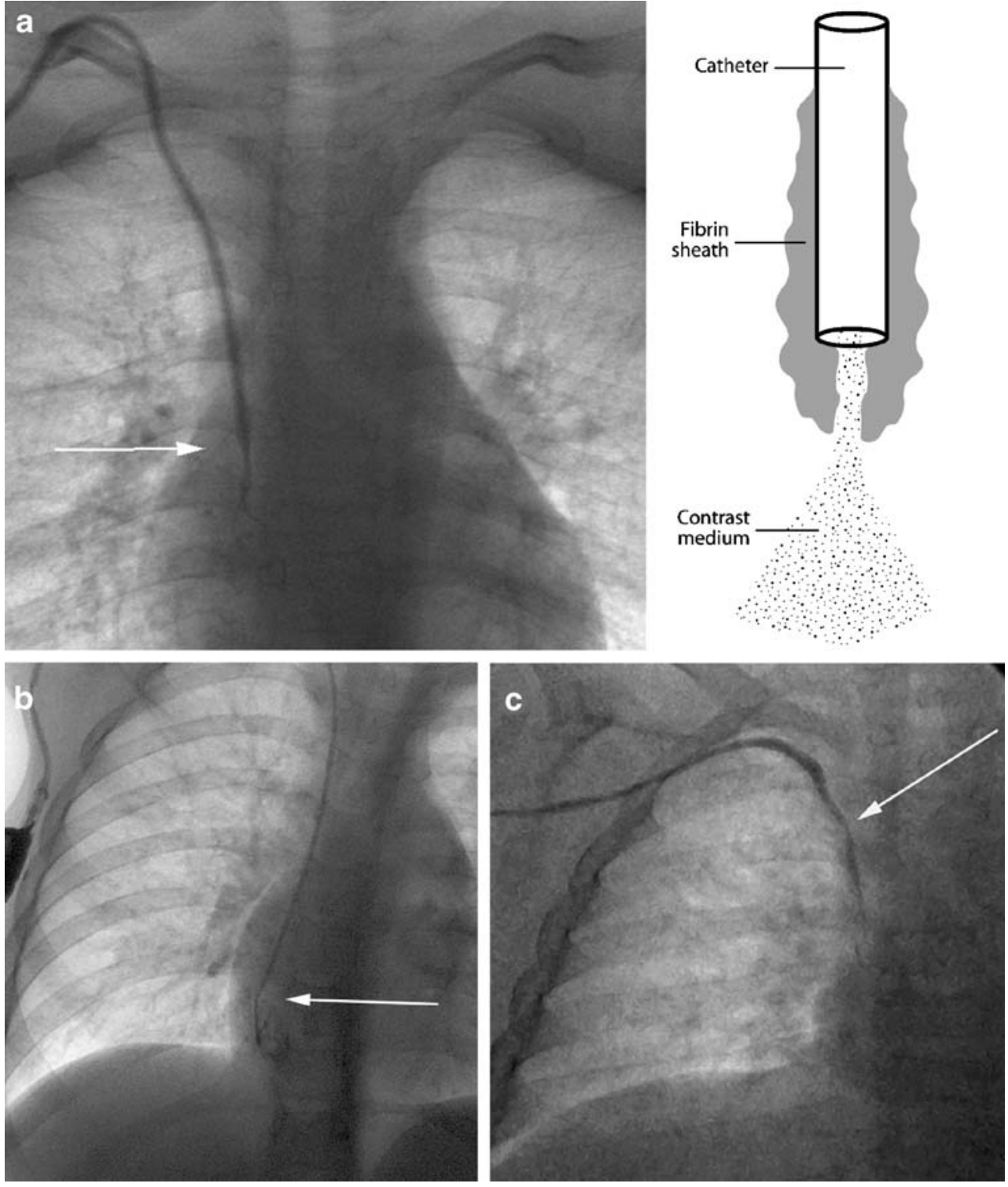

Kinks in the subcutaneous tunnel of a CVC can often be straightened by accessing either the venous access site or the skin exit site of the catheter. Intervention at the venous access site may result in an increase in the intravascular length of the catheter, a result that is beneficial in catheters that have migrated back into the tunnel or the neck.

Catheter tips that have flicked out of position can occasionally be repositioned using one of several techniques. Often a forceful injection of saline through the displaced catheter will encourage the tip to return to its original position, in the direction of flowing blood [10]. Obstinate catheters may respond to placement of a guidewire through the catheter lumen, which stiffens the catheter sufficiently to encourage repositioning of the catheter $[10,48]$. An alternative technique involves venous access via a femoral approach, allowing the displaced catheter to be snared and pulled back into the appropriate vessel [10, 49-51].

Catheter tip thrombus can be dislodged by inserting a tip deflecting guidewire through the catheter and rotating the wire once it is just beyond the tip of the catheter [9, 10]. Such techniques, although effective, are rarely long lasting.

Fibrin sheath stripping techniques can be attempted in patients in whom replacement of a malfunctioning catheter is undesirable, such as those with a high risk for general anaesthesia or limited alternative access sites. Again, femoral venous access is obtained and the catheter is ensnared using a standard vascular snare device (Fig. 28). Gentle manipulation of the snare along the length of the catheter is 


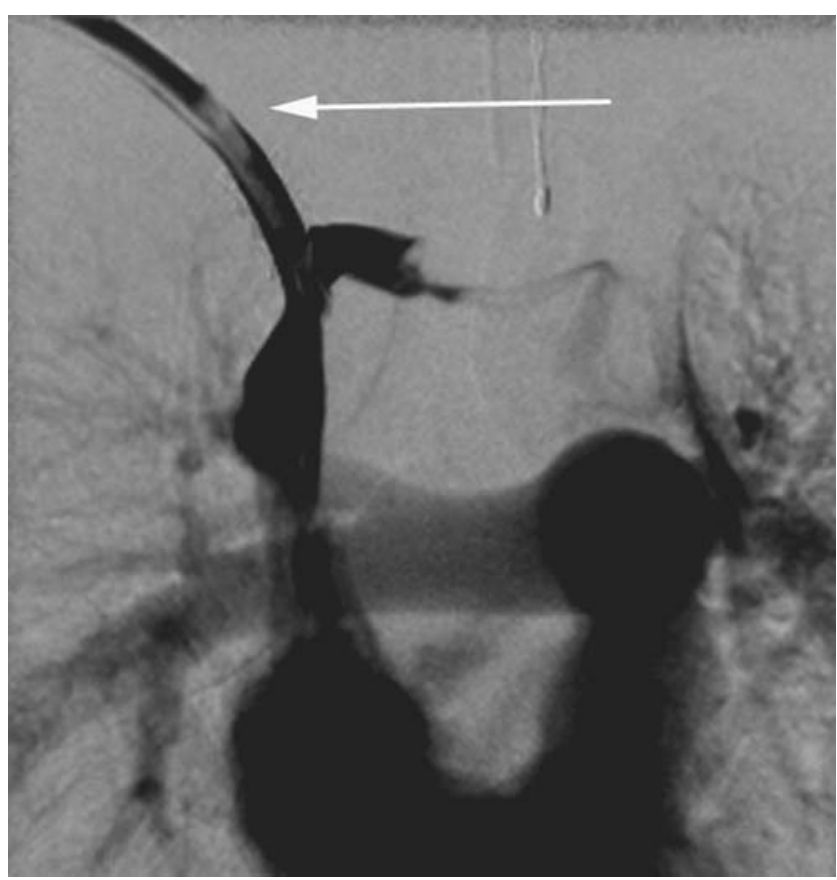

Fig. 23 Image acquired during contrast medium injection through one lumen of a double-lumen catheter in a 2-year-old female. There is a filling defect within the catheter (arrow) at the site of a focal intraluminal thrombus
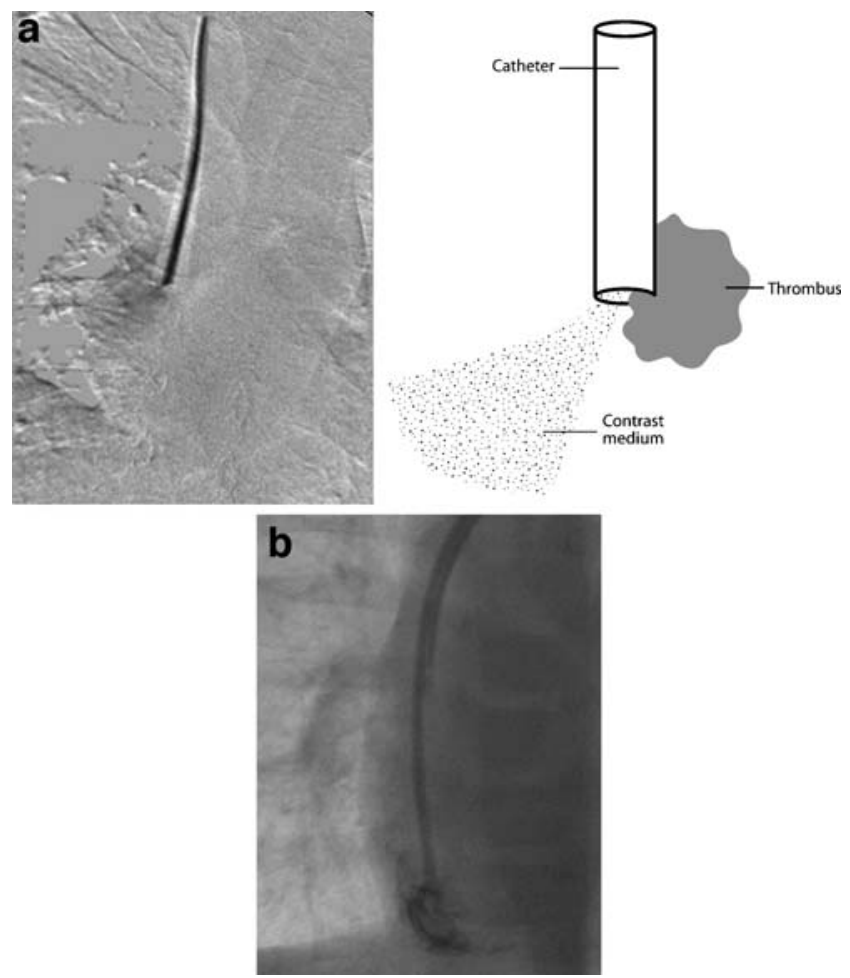

Fig. 24 Thrombus. a Fluoroscopic image acquired during a catheter contrast study in a 3-year-old female. The contrast medium jet is deflected away from the long axis of the catheter, implying the presence of partially occlusive thrombus at the catheter tip. The effect of the tip thrombus is shown diagrammatically. b Thrombus is adherent to the tip of the distal lumen of a haemodialysis catheter in a 7 -year-old male. Contrast medium exits the lumen eccentrically and outlines the thrombus

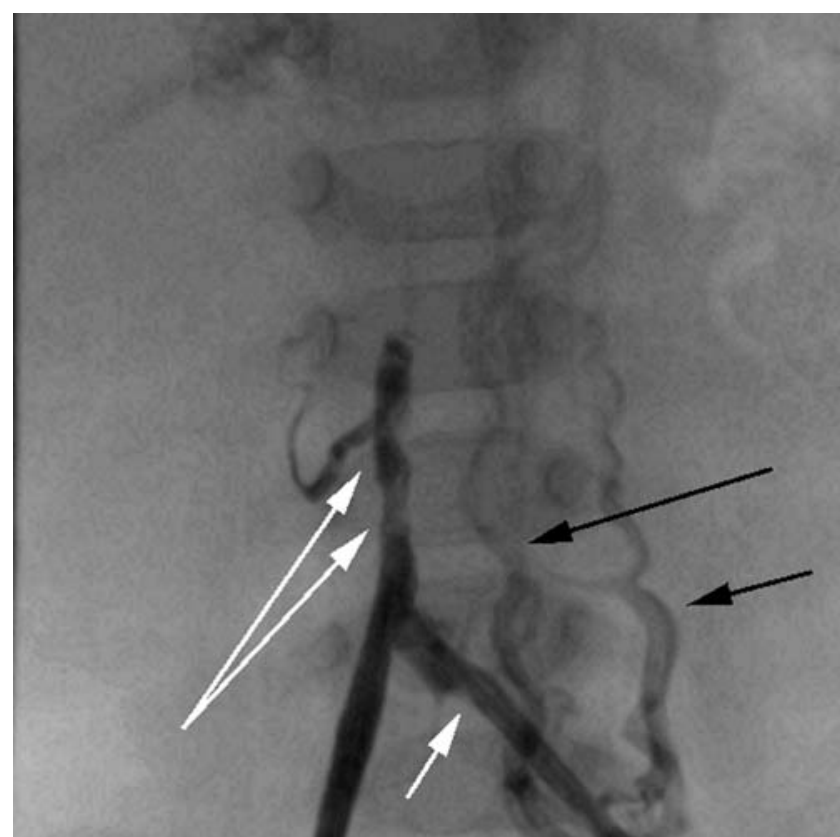

Fig. 25 Contrast study of a left femoral catheter in a 7-month-old child. The short white arrow shows the tip of the catheter. Contrast medium outlines the common iliac veins and IVC. There are several filling defects in the IVC (long white arrows) consistent with venous thrombus beyond the catheter tip. Note that contrast medium also refluxes into ascending lumbar veins on the left (black arrows)

performed in an attempt to dislodge and retrieve the fibrin sheath $[9,10,51-55]$. Symptomatic pulmonary embolism is unlikely, although the procedure is contraindicated in subjects with a right to left shunt or severe cardiopulmonary disease that will not tolerate a pulmonary embolus $[32,55]$.

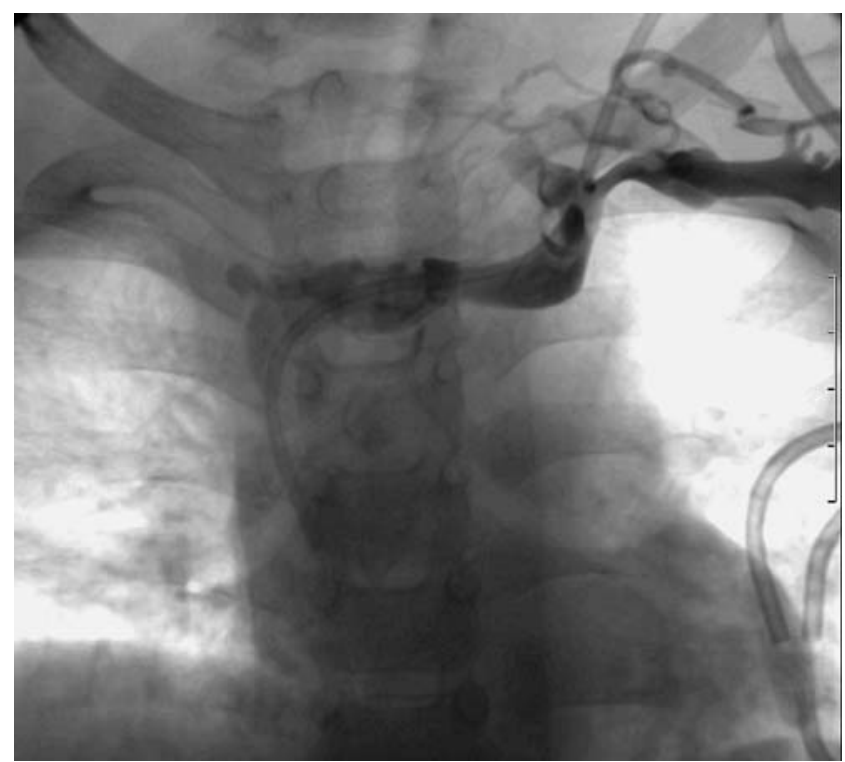

Fig. 26 Left arm venography in a 1-year-old female with a malfunctioning tunnelled catheter in situ. Contrast medium fails to fill the occluded SVC and there are irregular filling defects within the left brachiocephalic vein, consistent with mural thrombi. Small collaterals are forming in the left supraclavicular region 


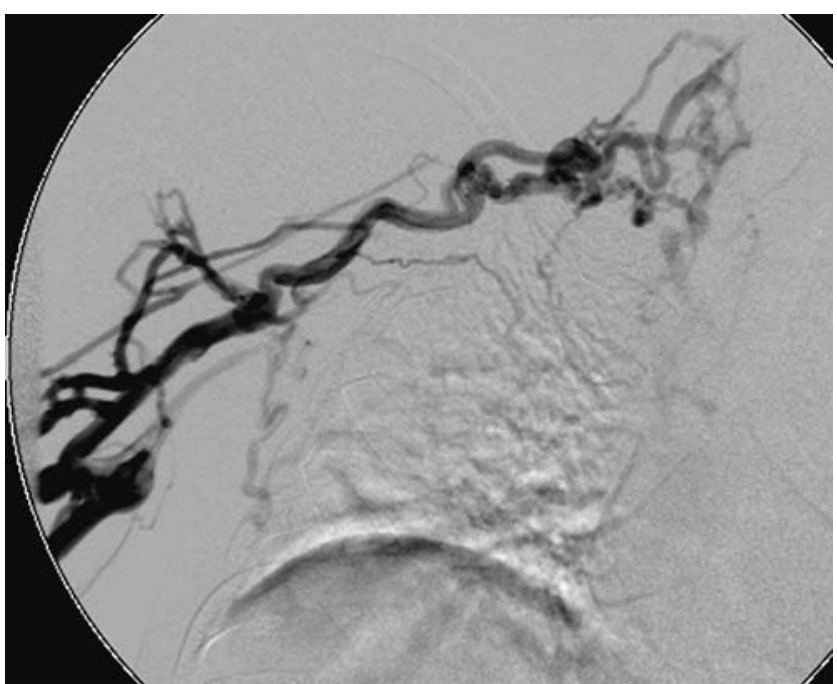

Fig. 27 Right arm venogram in an 8-year-old boy performed prior to placement of a new catheter. The study demonstrates multiple irregular venous collaterals draining the arm following occlusion of the subclavian and brachiocephalic veins

\section{Implications for catheter replacement techniques}

The results of catheter contrast medium studies are often valuable in planning catheter replacement. Operators are likely to alter their approach to venous access in the presence

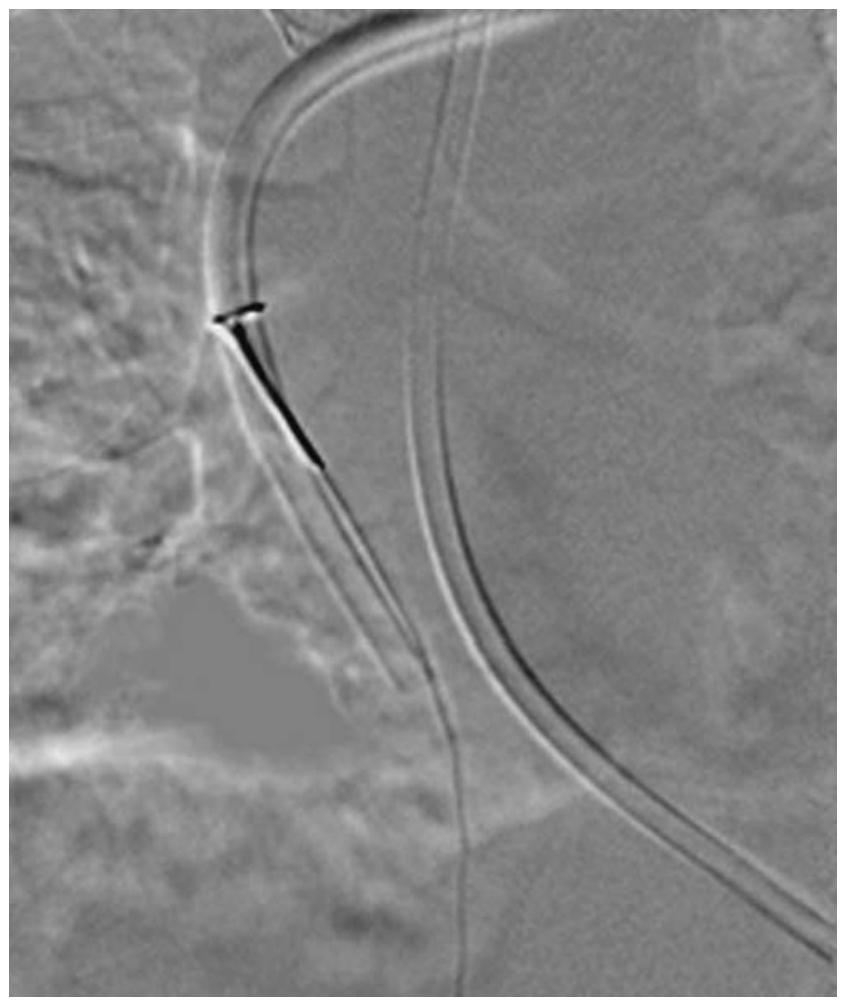

Fig. 28 The distal portion of a malfunctioning haemodialysis catheter has been snared from a femoral approach in an attempt to strip the fibrin sheath from the catheter

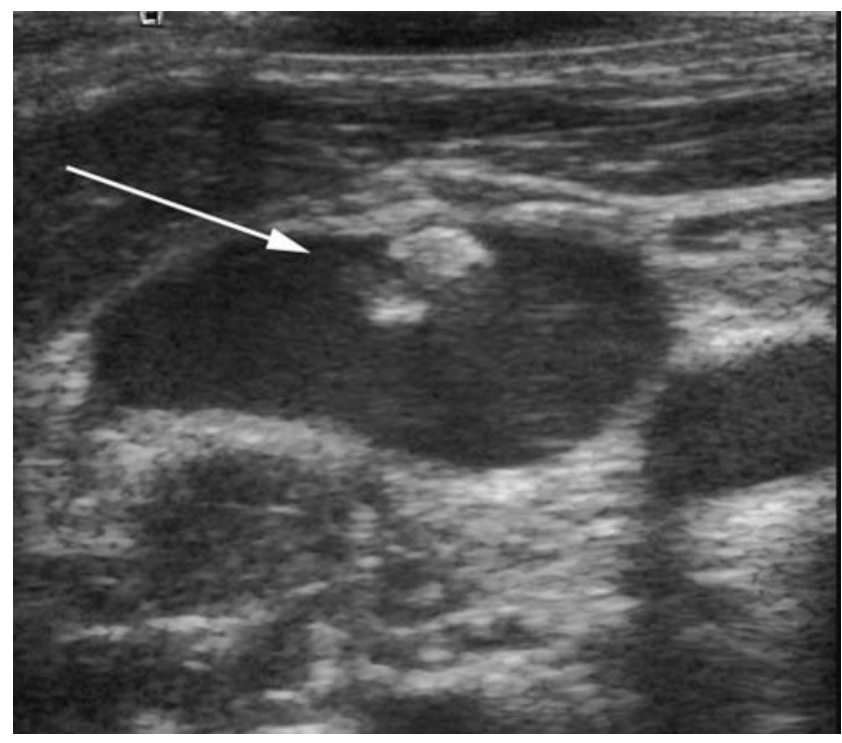

Fig. 29 US image of the right internal jugular vein in a 12-year-old female. A linear filling defect is attached to the vessel wall (arrow), likely to represent a previous fibrin sheath in this patient with a history of multiple previous access catheters

of a documented fibrin sheath or venous occlusion. Resiting a catheter into the lumen of a fibrin sheath is highly likely to result in a rapid reestablishment of the sheath and subsequent catheter occlusion. In cases where reaccess of a vessel containing a fibrin sheath is necessary, it is sensible to attempt an US-guided puncture parallel to the fibrin sheath, rather than into it (Fig. 29). Alternatively, the sheath can be deliberately cannulated so that a guidewire is placed through the sheath followed by a standard angioplasty balloon that, when inflated, encourages break-up of the sheath or incorporation of the sheath into the vessel wall $[9,53]$.

Documentation of complete venous occlusion is vital prior to replacement of a CVC. This allows a more considered approach to catheter placement and appropriate discussion with the family prior to a difficult procedure. Recanalization and venous stenting techniques or use of unusual access sites should be considered in children who would otherwise require open surgical procedures to maintain reliable venous access.

\section{Conclusion}

Reliable central venous access is key to the management of many paediatric conditions. The insertion of central venous access devices is increasingly becoming the remit of the interventional radiologist, a paradigm shift that will require general radiologists to play some role in the subsequent management of these catheters. In institutions where the specific cause of catheter malfunction may alter subsequent management, radiologists need to be familiar with the catheter types used and their imaging appearances, both on plain radiography 
and on contrast investigations. Accurate diagnosis of CVC malfunction can significantly alter outcome for patients in whom repeated venous access procedures can often become a source of greater morbidity than their primary condition.

\section{References}

1. Trerotola SO, Johnson MS, Harris VJ et al (1997) Outcome of tunneled hemodialysis catheters placed via the right internal jugular vein by interventional radiologists. Radiology 203:489-495

2. Mauro MA, Jaques PF (1997) Radiologic placement of long-term central venous catheters: a review. J Vasc Interv Radiol 4:127-137

3. Rush J, Baskin KM, Cahill AM et al (2005) Central venous access by different service providers: does it matter who puts in the port? (abstract). Pediatr Radiol 35 [Suppl 1]:S76

4. Roebuck DJ, Kleidon TM, McLaren CA et al (2005) Internal jugular vein (IJV) patency after central venous (CV) access (abstract). Pediatr Radiol 35 [Suppl 1]:S75

5. Blum AS (1999) The role of the interventional radiologist in central venous access. J Intraven Nurs 22 [Suppl 6]:S32-S39

6. McBride KD, Fisher R, Warnock N et al (1997) A comparative analysis of radiological and surgical placement of central venous catheters. Cardiovasc Intervent Radiol 20:17-22

7. Lamaris JS, Post PJ, Zonderland HM et al (1990) Percutaneous placement of Hickman catheters: comparison of sonographically guided and blind techniques. AJR 155:1097-1099

8. Lund GB, Trerotola SO, Scheel PF et al (1996) Outcome of tunneled hemodialysis catheters placed by radiologists. Radiology 198:467-472

9. Namyslowski J, Trerotola SO (2000) Interventional radiologic placement and management of infusion catheters. In: Savada SJ, Trerotola SO (eds) Venous interventional radiology with clinical perspectives. Thieme, New York, pp 325-346

10. Kidney DD, Nguyen DT, Deutsch LS (1988) Radiologic evaluation and management of malfunctioning long-term central vein catheters. AJR 171:1251-1257

11. Kim FM, Burrows PE, Hoffer FA et al (1996) Interpreting the results of pediatric central venous catheter studies. Radiographics $16: 747-754$

12. Boardman P, Hughes JP (1998) Radiological evaluation and management of malfunctioning central venous catheters. Clin Radiol 53:10-16

13. Garg M, Chang CC, Merritt RJ (1989) An unusual case presentation: pericardial tamponade complicating central venous catheter. J Perinatol 9:456-457

14. Rogers BB, Berns SD, Maynard EC et al (1990) Pericardial tamponade secondary to central venous catheterisation and hyperalimentation in a very low birthweight infant. Pediatr Pathol 10:819-823

15. Sasidharan P, Billman D, Heimler R et al (1996) Cardiac arrest in an extremely low birth weight infant: complication of percutaneous central venous catheter hyperalimentation. J Perinatol 16:123-126

16. Makwana N, Lander A, Buick R et al (2003) Unusual complication of a central venous catheter in a neonate. Arch Dis Child Fetal Neonatal Ed 88:F440

17. Leipala JA, Petaja J, Fellman V (2001) Perforation complications of percutaneous central venous catheters in very low birth weight infants. J Paediatr Child Health 37:168-171

18. Liossis G, Bardin C, Papageorgiou A (2003) Comparison of risks from percutaneous central venous catheters and peripheral lines in infants of extremely low birth weight: a cohort controlled study of infants $<1000$ g. J Matern Fetal Neonatal Med 13:171-174
19. Nowlen TT, Rosenthal GL, Johnson GL et al (2002) Pericardial effusion and tamponade in infants with central catheters. Pediatrics 110:137-142

20. Beardsall K, White DK, Pinto EM et al (2003) Pericardial effusion and cardiac tamponade as complications of neonatal long lines: are they really a problem? Arch Dis Child Fetal Neonatal Ed 88: F292-F295

21. Cadman A, Lawrence JA, Fitzsimmons L et al (2004) To clot or not to clot? That is the question in central venous catheters. Clin Radiol 59:349-355

22. Schutz JC, Patel AA, Clark TW et al (2004) Relationship between chest port catheter tip position and port malfunction after interventional radiologic placement. J Vasc Interv Radiol 15:581-587

23. Cohn DE, Mutch DG, Rader JS et al (2001) Factors predicting subcutaneous implanted central venous port function: the relationship between catheter tip location and port failure in patients with gynecologic malignancies. Gynecol Oncol 83:533-536

24. Petersen J, Delaney JH, Brakstad MT et al (1999) Silicone venous access devices positioned with their tips high in the superior vena cava are more likely to malfunction. Am J Surg 178:38-41

25. Lucas H, Attard-Montalto SP, Saha V et al (1996) Central venous catheter tip position and malfunction in a paediatric oncology unit. Pediatr Surg Int 11:159-163

26. Jimenez M, Baskin KM, Cahill AM et al (2005) The elusive cavoatrial junction: anatomic and biometric study of the superior vena cava (abstract). Pediatr Radiol 35 [Suppl 1]:S59

27. Yoon SZ, Shin JH, Hahn S (2005) Usefulness of the carina as a radiographic landmark for central venous catheter placement in paediatric patients. Br J Anaesth 95:514-517

28. Nazarian GK, Bjarnason H, Dietz CA et al (1997) Changes in tunneled catheter tip position when a patient is upright. J Vasc Intervent Radiol 8:437-441

29. Kowalski CM, Kaufman JA, Rivitz SM et al (1997) Migration of central venous catheters: implications for initial catheter tip positioning. J Vasc Intervent Radiol 8:443-447

30. Chang TC, Funaki B, Szymski GX (1998) Are routine chest radiographs necessary after image-guided placement of internal jugular central venous access devices? AJR 170:335-337

31. Garofalo RS, Zaleski GX, Lorenz JM et al (1999) Exchange of poorly functioning tunneled permanent hemodialysis catheters. AJR 173:155-158

32. Weschler RJ, Spirn PW Conant EF et al (1993) Thrombosis and infection caused by thoracic venous catheters: pathogenesis and imaging findings. AJR 160:467-471

33. Hoshal VL, Ause RG, Hoskins PA (1971) Fibrin sleeve formation on indwelling subclavian central venous catheters. Arch Surg 102:353-358

34. Namyslowski J (2002) Management of catheter-induced thrombosis. Tech Vasc Interv Radiol 5:85-88

35. Allen AW, Megargell JL, Brown DB et al (2000) Venous thrombosis associated with the placement of peripherally inserted central catheters. J Vasc Interv Radiol 11:1309-1314

36. Gonsalves GF, Eschelman DJ, Sullivan KL et al (2003) Incidence of central vein stenosis and occlusion following upper extremity PICC and port placement. Cardiovasc Intervent Radiol 26:123-127

37. Trerotola SO, Kuhn-Fulton J, Johnson MS et al (2000) Tunneled infusion catheters: increased incidence of symptomatic venous thrombosis after subclavian versus internal jugular access. Radiology 217:89-93

38. Oguzkurt L, Tercan F, Torun D et al (2004) Impact of short-term hemodialysis catheters on the central veins: a catheter venographic study. Eur J Radiol 52:293-299

39. Chait P, Verbeeteb K, Amaral J et al (2005) Central venous catheter $(\mathrm{CVC})$ related thrombosis in haematology-oncology pediatric population (abstract). Pediatr Radiol 35 [Suppl 1]:S79 
40. Chesler L, Feusner JH (2002) Use of tissue plasminogen activator (rt-PA) in young children with cancer and dysfunctional central venous catheters. J Pediatr Hematol 24:653-656

41. Whigham CJ, Lindsey JI, Goodman CJ et al (2002) Venous port salvage utilizing low dose tPA. Cardiovasc Intervent Radiol 25:513-516

42. Santilli J (2002) Fibrin sheaths and central venous catheter occlusions: diagnosis and management. Tech Vasc Interv Radiol 5:89-94

43. Savader SJ, Ehrman KO, Porter DJ et al (2001) Treatment of hemodialysis catheter-associated fibrin sheaths by rt-PA infusion: critical analysis of 124 procedures. J Vasc Interv Radiol 12:711-715

44. Semba CP, Deitcher SR, Li X et al (2002) Treatment of occluded central venous catheters with alteplase: results in 1,064 patients. J Vasc Interv Radiol 13:1199-1205

45. Liu CY, Jain V, Shields AF et al (2004) Efficacy and safety of reteplase for central venous catheter occlusion in patients with cancer. J Vasc Interv Radiol 15:39-44

46. Kaufman JA, Fan CM, Geller SC et al (1997) Percutaneous revision of excess length from an implanted long-term central venous access device. AJR 169:1732-1734

47. Murthy R, Arbabzadeh M, Richard H et al (2004) Endovascular technique for revision of excess catheter length in subcutaneous implanted venous access devices. Cardiovasc Intervent Radiol $27: 259-261$

48. Lois JF, Gomes AS, Pusey E (1987) Non-surgical repositioning of central venous catheters. Radiology 156:329-333
49. Thalhammer A, Jacobi V, Balzer J et al (2002) Repositioning of malpositioned or flipped central venous catheters. Eur Radiol 12:698-700

50. Hartnell GG, Gates J, Suojanen JN et al (1996) Transfemoral repositioning of malpositioned central venous catheters. Cardiovasc Interv Radiol 19:329-331

51. Bessoud B, de Baere T, Kuoch Y et al (2003) Experience at a single institution with endovascular treatment of mechanical complications caused by implanted central venous access devices in pediatric and adult patients. AJR 180:527-532

52. Angle JF, Shilling AT, Schenk WG et al (2002) Utility of percutaneous intervention in the management of tunnelled hemodialysis catheters. Cardiovasc Intervent Radiol 26:9-18

53. Janne d'Othee B, Tham JC, Sheiman RG (2006) Restoration of patency in failing tunnelled haemodialysis catheters: a comparison of catheter exchange, exchange and balloon disruption of the fibrin sheath, and femoral stripping. $J$ Vasc Interv Radiol 17:1011-1015

54. Grey RJ, Levitin A, Buck D et al (2000) Percutaneous sheath stripping versus transcatheter urokinase infusion for malfunctioning well-positioned tunneled central venous dialysis catheters: a prospective, randomized trial. J Vasc Interv Radiol 11:11211129

55. Crain MR, Mewissen MW, Ostrowski GJ et al (1996) Fibrin sleeve stripping for salvage of failing hemodialysis catheters: technique and initial results. Radiology 198:41-44 